\title{
Famennium-Anthozoa aus Marokko. 1. Czarnockia Różkowska, 1969 (Rugosa)
}

\author{
Dieter Weyer ${ }^{1}$ \\ Mit 7 Abbildungen und 3 Tafeln
}

\section{Zusammenfassung}

Aus Cephalopoda-Kalken der mittleren/oberen Clymenia-Stufe (Niveau mit Gonioclymenia) des Tafilalt im Anti-Atlas ist Czarnockia maroccana n. sp. beschrieben. Die Kollektion von 7 Polyparen zeigt eine erhebliche innerartliche Variabilität, die nur mit ausführlichen Schliffserien aller subtabularen und calicularen Regionen erfassbar wird. Das seltene, bisher allein aus der Wocklumeria-Stufe des Heiligkreuz-Gebirges in Polen bekannte Petraiidae-Genus - Endglied einer phylogenetischen Reihe Neaxon $\rightarrow$ Petraia $\rightarrow$ Famennelasma $\rightarrow$ Czarnockia - ist erstmals in einem weiteren Verbreitungsareal nachgewiesen, dürfte aber kosmopolitisch in der Psychrosphäre (Cyathaxonia-Fazies) des Ober-Famennium anzutreffen sein.

Schlüsselwörter: ahermatypische Korallen, Cyathaxoniina-Taxonomie, Tafilalt (Marokko).

\author{
Summary \\ Famennian Anthozoa from Morocco. 1. Czarnockia Różkowska, 1969 (Rugosa)
}

The new species Czarnockia maroccana is described from cephalopod limestones of the middle/upper Clymenia genozone (level with Gonioclymenia) in the Tafilalt district of the Anti Atlas region. The small collection of seven syntypes demonstrates a surprising intraspecific variation visible only after intensive serial sectioning of all subtabular and calicular parts of the skeleton. This is the first record of the rare genus from a second area outside the type occurrence in the Wocklumeria genozone of the Polish Holy Cross Mountains. As a member of the phyletic line Neaxon $\rightarrow$ Petraia $\rightarrow$ Famennelasma $\rightarrow$ Czarnockia, the taxon should be found cosmopolitically in the psychrospheric Cyathaxonia facies of Upper Famennian times.

Key words: ahermatypic corals, taxonomy of Cyathaxoniina, Tafilalt (Morocco).

\section{Einleitung}

Lange Zeit galt das jüngere Oberdevon als eine Krisenzeit für paläozoische Korallen, die nach dem globalen Riffsterben an der Frasnium/Famennium-Grenze (Oberer Kellwasser-Event) nur noch selten beobachtet und untersucht wurden (Sorauf \& Pedder 1986). Hermatypische Anthozoa-Faunen der Flachwasser-Fazies standen bei diesem Urteil im Vordergrund, wobei EtroeungtKorallen früher oft als basales Unterkarbon datiert waren - inzwischen sind einige Vorkommen im Ober-Famennium besser bekannt:

Westeuropa (Frech 1885, Poty 1984)

Russland (Vasilyuk 1970; Novaya Zemlya Gorskiy 1938, Kuznezk-Becken - Dobrolyubova \& Kabakovich 1966, Ural - Gorskiy et al. 1975, Omolon-Gebirge - Onoprienko 1979)

USA (Sorauf 1992)

China (Wu et al. 1981, Lin \& Xu 1994, Poty \& Xu 1996; Umdatierung der klassischen ältesten Unterkarbon-Korallenzone mit Cystophrentis als oberstes Famennium - Yu 1931, Wang 1987)

Polen (Berkowski 2001 - erster zweifelsfreier Nachweis von Phillipsastraeidae im Ober-Famennium)

Die taxonomische Vielfalt der in der Regel seltenen und überall meist vernachlässigten ahermatypischen Korallen der Psychrosphäre hat für das Famennium zum ersten Mal Różkowska (1969) anhand überreicher Kollektionen aus dem polnischen Heiligkreuz-Gebirge gezeigt. Ähnlich di-

1 Löwestr. 15, D-10249 Berlin, Germany. Eingegangen March, angenommen July 2002 
verse Assoziationen wurden auch in der Cephalopoden-Fazies Deutschlands (hauptsächlich Thüringisches Schiefergebirge) nachgewiesen (Weyer 1984). Das dritte markante Fundgebiet ist NordChina (Liao \& Cai 1987, Guo 1990, Soto \& Lin 1997, 2000, Soto \& Liao 2002). Über den Kontrast solcher Kaltwasser-Gemeinschaften an der Frasnium/Famennium-Grenze sind noch keine Aussagen möglich, weil weltweit derartige Faunen des Frasnium fast unbekannt blieben.

Hinzu kommt jetzt als vierte Region der Welt mit reichen Faunen der Cyathaxonia-Fazies (sensu Hill 1938) das Famennium von Marokko (vor allem Tafilalt und Maider im Anti-Atlas). Korallen-Funde (Rugosa, Heterocorallia, Tabulata) wurden schon vereinzelt in Faunenlisten erwähnt (beispielsweise Lecointre 1926: 36, 124; Tourneur 1994: 4) und morphologisch/taxonomisch beschrieben (Termier \& Termier 1950: 75, 99; Weyer 1995, 1997). Eigene Aufsammlungen (1995, 2000, 2001) sowie freundlicherweise von Fachkollegen (Prof. Dr. R. T. Becker, Münster, J. Bockwinkel, Leverkusen, Dr. V. Ebbighausen, Odenthal, Dr. C. Klug, Tübingen, Dr. D. Korn, Tübingen, Dr. P. Sartenaer, Bruxelles) überlassene Einzelfunde repräsentieren ein umfangreiches Anthozoa-Material aus dem stratigraphischen Intervall Cheiloceras-Stufe bis Wocklumeria-Stufe (Schwerpunkt Clymenia-Stufe).

Das hier behandelte Genus Czarnockia gehört (ebenso wie Guerichiphyllum Różkowska, 1969, sensu lato) innerhalb ahermatypischer Taxa zu den in der Psychrosphäre des Famennium generell seltenen pleonophoren Bauplänen (im Sinne des Glossars von Dybowski 1873). Solche Dissepiment-tragenden Formenkreise existieren gleichfalls untergeordnet - auch in den meisten unterkarbonischen Faunen der Cyathaxonia-Fazies: Caninia Michelin, 1840, Rylstonia Hudson \& Platt, 1927, Hebukophyllum Liao \& Cai, 1987. Bei Czarnockia besteht allerdings die Besonderheit, dass Dissepimente erst spät adult erscheinen, was eine Abgrenzung der Gattung gegen ihren nur diaphragmatophoren Famennium-Vorläufer Famennelasma Weyer, 1973 erschwert (zumal mittlere und distale Kelchbereiche dieser kleinen Einzelkorallen auch heutzutage oft gar nicht untersucht werden). Die sieben verfügbaren, mit insgesamt 99 Querschliffen und 19 Längsschliffen ontogenetisch ausführlich untersuchten Polypare der neuen marokkanischen Czarnockia-Art demonstrieren mit ihrer in diesem Ausmaß unerwarteten beachtlichen Variationsbreite eindringlich, wie konventionelle Stu- dien (beispielsweise aus China) anhand weniger Schliffe zu taxonomischen Fehlurteilen führen müssen (Zuordnung zu mehreren Taxa) oder kaum $\mathrm{zu}$ beurteilende, nicht wiedererkennbare Arten aufstellten (Czarnockia simplex Różkowska, 1969, Friedbergia sinensis Guo, 1990, Famennelasma sinense Guo, 1990, Friedbergia bipartita Różkowska, 1969 sensu Soto \& Lin 2000).

Die fazielle Nomenklatur bei Hill (1938: 5-12) bietet aus heutiger Sicht rein formale, jedoch zu beachtende Widersprüche. Ihre Caninid-Clisiophyllid Fauna bezieht sich ausdrücklich nicht auf die ahermatypische echte Caninia, sondern auf heute generisch separierte hermatypische caniniide Taxa wie Siphonophyllia Scouler in McCoy, 1844, Bothrophyllum Trautschold, 1879, Caninophyllum Lewis, 1929 und Haplolas. ma Semenoff-Tian-Chansky, 1974. Ihr Terminus Cyathaxonia Fauna (kommentiert in Kullmann 1997) wurde von Oliver (1992: 139) für Devon-Assoziationen (nur im Famennium mit Cyathaxonia) in Laccophyllid assemblage geändert, aber ich bevorzuge in Anlehnung an den ursprünglichen Vorschlag von Hill (1939) die Bezeichnung Cyathaxoniidae-Fauna/Fazies/Assoziation, weil Laccophyllum Simpson, 1900 als direkter Vorfahr von Cyathaxonia Michelin, 1847 in die gleiche Familie (als eigene Subfamilia) zu gruppieren ist (Weyer 2001: 73)

Für die extrem seltene, bisher nur aus Polen (Heiligkreuz-Gebirge) gemeldete Gattung Czarnockia ist jetzt mit Marokko (Tafilalt) ein zweites, Gondwana-nah weit entferntes Fundareal belegt. Paläogeographisch vermittelnde Vorkommen werden aber in Mittel- und Westeuropa (Thüringisches und Rheinisches Schiefergebirge, Montagne Noire) erwartet und könnten sich unter reichlich vorliegenden, noch nicht ausführlich präparierten Famennelasma-Kollektionen verbergen. Erfahrungsgemäß waren solche ahermatypischen Genera auch im Famennium nahezu kosmopolitisch verbreitet.

Das Rugosa-Material wird im Museum für Naturkunde der Humboldt-Universität zu Berlin aufbewahrt. In den Querschliff-Illustrationen sind traditionell 6 Protosepten markiert; alle Zeichnungen (nicht aber die Fotos) wurden seitenrichtig orientiert (Kelcheinblick von oben). Vereinzelt wurden Tabulae-Schnitte in Querschliff-Zeichnungen nur als dünne Basallinien ohne ihre stereoplasmatischen Verdickungen gezeichnet.

Verwendete Abkürzungen bei der Präparation: CS $=$ Querschliff (cross section), LS = Längsschliff (longitudinal section), $\mathrm{P}=$ Lackfilm (peel), $\mathrm{R}=$ Reststück, TS $=$ Dünnschliff (thin section). In den Septenformeln bedeuten: $\mathrm{D}=$ Durchmesser (in $\mathrm{mm}$ ), $\mathbf{n}=$ Anzahl der Großsepten, $\mathbf{N}=$ Anzahl aller Septen (Groß- + Kleinsepten, ohne Septen dritter Ordnung). 


\section{Supraordo Rugosa Milne-Edwards \& Haime Ordo Stauriida Verrill \\ Subordo Cyathaxoniina Spasskiy \\ Familia Petraiidae Koninck}

\section{Genus Czarnockia Różkowska, 1969}

Das Taxon ist in der emendierten Fassung von Weyer (2000: Revision des Holotypus der Typusart Czarnockia obliqua Różkowska, 1969 aus der Wocklumeria-Stufe von Kowala im Heiligkreuz-Gebirge, Polen) als Endglied einer phylogenetischen Reihe Neaxon $\rightarrow$ Petraia $\rightarrow$ Famennelasma $\rightarrow$ Czarnockia interpretiert.

\section{Czarnockia maroccana n. sp.}

Abb. 1-7, Tafel 1-3

2000 Czarnockia sp. n. aff. obliqua Różkowska 1969 Weyer: 87, Abb. 1/1-4.

Holotypus: Polypar Nr. MB.-K.655. $=16$ CS (1 TS, 15 P) + 9 LS (P) + 8 R. (Kollektion D. Weyer 1995, Original zu Weyer 2000: dort irrtümlich als Nr. MB.-K.629. nummeriert). - Abb. 1A-P, Tafel 1: 1-6.

Locus typicus: El Atrous (Nord) - km-lange Schürfe des Bergbaus auf Fossilien des Gonioclymenia-Niveaus am Nordrand der Unterkarbon-Synklinale (nordwestlich vom Brunnen El Atrous, ca. $14 \mathrm{~km} \mathrm{NW} \mathrm{Taouz).} \mathrm{Der} \mathrm{Fundort} \mathrm{ist} \mathrm{er-}$ wähnt bei Becker (1993: 139 - nur Profilteil Ober-Frasnium/ Unter-Famennium) und Korn et al. (2000: 72, Abb. 3 - El Atrous North).

Stratum typicum: Ober-Famennium, mittlerer bis oberer Teil der Clymenia-Stufe (doV, Schichten mit Gonioclymenia).

Die Korallen-Kollektion wurde im hinterlassenen losen Schutt des Gonioclymenia-Fossilbergbaus geborgen. Eine präzise Datierung ist trotzdem möglich, weil oft typische Gonioclymenia-Fragmente in denselben Gesteinsbrocken enthalten waren.

Weiterer Fundort: Ouidane Chebbi (Südost), ca. $46 \mathrm{~km}$ ESE Erfoud. Mittlere/obere Clymenia-Stufe Schichten mit Gonioclymenia). Das Profil (Ober-Famennium, Devon/Karbon-Grenze) ist erwähnt bei Belka et al. (1999: Abb. 2, 3, Ouidane Chebbi III) und Korn (1999: 149, Abb. 4).

Paratypen: 6 Polypare aus eigenen Aufsammlungen 1995, vom locus typicus (Nr. K.656.-K.660.) und vom Zweitfundort Ouidane Chebbi Südost (Nr. K.661.).

Nr. MB.-K.656. 16 CS $(3$ TS, 13 P) +2 LS $(P)+5$ R (Abb. 2A-M, Tafel 1: 7-10).

Nr. MB.-K.657. 17 CS (1 TS, 16 P) +4 LS $(\mathrm{P})+8$ R (Abb. 3A-L, Tafel 3: 5-6).

Nr. MB.-K.658. 10 CS $(1$ TS, 9 P) +2 LS $(P)+6$ R (Abb. 4A-G, Tafel 3: 7).

Nr. MB.-K.659. 10 CS (2 TS, 8 P) +2 LS $(P)+5$ R (Abb. 5A-G, Tafel 2: 7-8).

Nr. MB.-K.660. 14 CS (2 TS, 12 P) +4 R (Abb. 6A-F, Tafel 3: $1-4)$.

Nr. MB.-K.661. 16 CS (2 TS, 14 P) + 3 R (Abb.7A-L, Tafel 2: $1-6)$.

Diagnose: Czarnockia-Art mit relativ dünnen, radial stehenden Großsepten und nur calicular verkürztem Cardinalseptum, mit einzelnen normalen Dissepimenten im unteren und mittleren Kelch sowie mit wenigen lonsdaleioiden Dissepi- menten im oberen Kelch, und mit einfachem Tabularium aus Tabulae und untergeordnet Tabellae.

Beschreibung: Die breitkonischen Polypare haben bei Längen von $20-32 \mathrm{~mm}$ Kelchdurchmesser um 17-25 mm und sind in der Regel nur sehr leicht cornut, wobei das Cardinalseptum an der konvexen Seite liegt. Die Krümmung ist mitunter nur durch den stets deutlichen, kleinen oder großen Anheftungstalon bedingt und kann sich im Alter verlieren. Die Archaeotheca trägt zarte und gröbere wulstige Anwachsrugae sowie meist sehr schwache longitudinale Interseptalrippen, die manchmal auch weitgehend fehlen. Trotz der Präsenz von Septen dritter Ordnung wurden keine externen Septalfurchen von Hyposepten beobachtet, obwohl sie bei verwandten Taxa wie Famennelasma conus (Różkowska, 1969) vorkommen. Distal traten vereinzelt rejuvenescence-Einschnürungen auf (Tafel 2: 7, oberhalb von Abb. 4A). Die Kelchtiefe beträgt $50-60 \%$ der Polyparlänge.

Der adulte Septalapparat besteht aus 27-32 Großsepten und 19-24 Kleinsepten, die normal am Kelchrand ununterscheidbar als winzige Wandvorsprünge starten. Etwas tiefer im oberen Kelch beginnt die erste Längendifferenzierung; im mittleren Kelch werden die Großsepten allmählich länger (mit Ausnahme des deutlich verkürzten Cardinalseptums und der üblicherweise fast wie Kleinsepten wirkenden letzten Metasepten der vier Quadranten - Abb. 1E-G, 2D-F). Die Großsepten erreichen im unteren Kelch ihre maximale Länge von etwa $33 \%$ des Polyparlumens; zentral verbleibt ein großer septenfreier Raum. Ein Großseptum zeigt im Querschliffbild meist einen kleinen dreieckigen Sockel an der Wand, einen besonders dünnen mittleren Bereich und am Axialende eine ganz leichte bis deutliche Verdickung. An der Kelchbasis wächst das Cardinalseptum auf normale Großseptenlänge und verliert seine in einer Fossula gelegene Verkürzung, die subtabular nicht mehr erkennbar bleibt. Die anderen Protosepten unterscheiden sich nicht von den Metasepten und sind lediglich anhand der Septeninsertionsstellen (gemäß dem Kunth'schen Gesetz), der in Serienschliffen ermittelten Ontogenese und des Trends zur Bildung eines leicht biformen Tabulariums zu erkennen. Nur einmal (Abb. 7) ist das Antiseptum im unteren Kelch ganz leicht verlängert. Die vorübergehende Verkürzung des linken Antilateralseptums bei diesem Paratypus ist offensichtlich eine Wachstumsanomalie. Die Großsepten 
sind im Wesentlichen radial gestellt; eine leicht pinnate Anordnung tritt manchmal vorübergehend auf (bei Septeninsertionen, speziell an der Cardinalfossula - Abb. 2, 6).

Kurze Kleinsepten sind adult gut und überall gleichmäßig entwickelt. Es gibt keine Tendenz zur Bildung einer antiseptalen Triade und nicht die geringsten Anklänge an einen contratingenten Baustil. Auf Jugendstadien sind die Kleinsepten nicht mehr aktiv, sondern sekundär in der sich verdickenden Wand verschwunden. Der Zeitpunkt des aktiven Erscheinens wechselt beachtlich zwischen 5-12 mm D.

Die Ontogenie des Septalapparats konnte zwischen $1.7 \mathrm{~mm}$ und $25 \mathrm{~mm} \mathrm{D}$ ermittelt werden. Die betreffenden Septenformeln sind in den Ab- bildungen notiert. Im Prinzip lassen sich die typischen Stadien der phylogenetischen Reihe Nea$x o n \rightarrow$ Petraia $\rightarrow$ Famennelasma $\rightarrow$ Czarnockia gut erkennen. Die an der Kelchbasis erreichte finale Verdickung der Skelettelemente erfolgt während des gesamten Wachstums beinahe gleichmäßig (Abb. 1, 2, 7) oder war in der Jugend wesentlich kräftiger ausgeprägt (Abb. 3,5).

Septen dritter Ordnung vom Typ Meiosepten (Weyer 1984: 6, Tafel 7, Figs 14, 15) sind nahe am Kelchoberrand eines Paratypus markant ausgebildet (Abb. 2A, B). Bei zwei weiteren Exemplaren (Abb. 4A, 7A-F) erkennt man sie nur vereinzelt (wahrscheinlich wegen eines grundsätzlich spinösen Baustils). Das Stadium von Abb. 3A (4 untere Septen) unmittelbar am

\section{Legenden zu den Abbildungen 1 und 2}

Abb. 1. Czarnockia maroccana n. sp., Holotypus Nr. MB.-K.655., Clymenia-Stufe, El Atrous Nord. A, B. distale Querschliffe (Nr. 1, 2, mit lonsdaleioiden und einfachen Dissepimenten, Cardinalseptum unten in B). C. distaler Längsschliff (Nr. 3, mit 2 Dissepimentblasen). D. tangentialer Längsschliff im distalen Kelch (Nr. 7, 2 Großsepten und 1 Kleinseptum mit Septalrandspinae $=$ Trabekel) . E-H. caliculare Querschliffe (Nr. 10-13). I-K. Querschliffe der Kelchbasis (Nr. 14-16, caliculare Lumina punktiert). L, M. subtabulare mediane Längsschliffe (Nr. 17, 19, parallel zur Antiseptum/Cardinalseptum-Ebene). N-P. subtabulare Querschliffe (Nr. 20, 22, 24). × $3(\mathrm{E}, \mathrm{F}), \times 3.5(\mathrm{G}-\mathrm{I}), \times 4(\mathrm{~B}, \mathrm{~J}-\mathrm{M}), \times 6(\mathrm{~A}, \mathrm{~N}), \times 8(\mathrm{C}, \mathrm{D}, \mathrm{O})$ und $\times 12(\mathrm{P})$. Polypar- und Schliff-Fotos siehe Tafel 1: 1-6. Septenformeln:

\begin{tabular}{|c|c|c|c|c|c|c|c|}
\hline \multirow[t]{2}{*}{ Nr. K.655 } & $3 \mid 3$ & $3 \mid 3$ & \begin{tabular}{l|l}
4 & 4
\end{tabular} & $4 \mid 4$ & \begin{tabular}{l|l}
5 & 4
\end{tabular} & $5 \mid 5$ & $5 \mid 5$ \\
\hline & \begin{tabular}{l|l}
4 & 4
\end{tabular} & \begin{tabular}{l|l}
5 & 4
\end{tabular} & $5: 5$ & \begin{tabular}{l|l}
6 & 6
\end{tabular} & \begin{tabular}{l|l}
6 & 6
\end{tabular} & \begin{tabular}{l|l}
6 & 6
\end{tabular} & \begin{tabular}{l|l}
6 & 7
\end{tabular} \\
\hline n & 18 & 19 & 22 & 24 & 25 & 26 & 27 \\
\hline $\mathbf{N}$ & 28 & 30 & 36 & 41 & 42 & $44-45$ & 46 \\
\hline $\mathrm{D} \mathrm{mm}$ & 3.1 & 5.0 & 7.1 & 13.0 & 13.5 & $13.7-16.7$ & 18.0 \\
\hline Abb. & $1 \mathrm{P}$ & 10 & $1 \mathrm{~N}$ & $1 \mathrm{~K}$ & $1 . J$ & 1I, H, G, F, E & $\begin{array}{c}\text { Weyer } 2000 \\
\text { Abb. } 1 / 4\end{array}$ \\
\hline
\end{tabular}

Fig. 1. Czarnockia maroccana n. sp., holotype no. MB.-K.655., Clymenia genozone, El Atrous North. A, B. distal cross sections (no. 1, 2, with lonsdaleioid and simple dissepiments,cardinal septum of $\mathbf{B}$ at bottom). C. distal longitudinal section (no. 3 , with 2 dissepiments). D. tangential longitudinal sections within the distal calice (no. 7,2 major septa and 1 minor septum, with trabecular spines at the septal margins). $\mathbf{E}-\mathbf{H}$. calicular cross sections (no. 10-13). I-K. cross sections of the calicular base (no. 14-16, with stippled calicular lumina). L, M. median subtabular longitudinal sections (no. 17, 19, parallel to the plain of antiseptum and cardinal septum). N-P. subtabular cross sections (no. 20, 22, 24). × $3(\mathrm{E}, \mathrm{F}), \times 3.5(\mathrm{G}-\mathrm{I}), \times 4(\mathrm{~B}, \mathrm{~J}-\mathrm{M}), \times 6$ $(A, N), \times 8(C, D, O)$, and $\times 12(P)$. Photos of corallum and sections see pl. 1: 1-6. Septal formulae.

Abb. 2. Czarnockia maroccana n. sp., Paratypus Nr. MB.-K.656., Clymenia-Stufe, El Atrous Nord. A. distaler Querschliff (Nr. 1, mit 2 lonsdaleioiden Dissepimenten; Detailskizze von Antiseptum und rechtem Antilateralseptum, mit zugehörigen 2 Kleinsepten und 3 Septen 3. Ordnung). B-G. caliculare Querschliffe ( $\mathrm{Nr} .2,5,7-10$, in B-E mit einfachen Dissepimenten). H, I. mediane Längsschliffe der Kelchbasis (Nr. 11, 12, parallel zur Antiseptum/Cardinalseptum-Ebene, Antiseptum links). J. Querschliff der tiefsten Kelchbasis (Nr. 13, mit nur noch 1 calicularen Lumen am Cardinalseptum). K-M. subtabulare Querschliffe (Nr. 16-18). $\times 2(\mathrm{~A}$, Septendetail $\times 9.25), \times 2.5(\mathrm{~B}), \times 3(\mathrm{C}), \times 3.5(\mathrm{D}-\mathrm{F}), \times 4(\mathrm{G}-\mathrm{I}), \times 6(\mathrm{~J})$ und $\times 8(\mathrm{~K}-\mathrm{M})$. Polyparund Querschliff-Fotos siehe Tafel 1: 7-10. Septenformeln:

\begin{tabular}{|c|c|c|c|c|c|c|c|}
\hline Nr. K.656 & $2 \mid 2$ & \begin{tabular}{l|l}
3 & 3 \\
\end{tabular} & \begin{tabular}{l|l}
4 & 4 \\
\end{tabular} & \begin{tabular}{l|l}
4 & 4 \\
\end{tabular} & & \begin{tabular}{l|l}
5 & 4 \\
\end{tabular} & \\
\hline & \begin{tabular}{l|l}
5 & 5
\end{tabular} & \begin{tabular}{l|l}
5 & 5
\end{tabular} & \begin{tabular}{l|l}
6 & 6
\end{tabular} & \begin{tabular}{l|l}
7 & 6
\end{tabular} & $7 \mid 7$ & \begin{tabular}{l|l}
8 & 7
\end{tabular} & \begin{tabular}{l|l}
8 & 8
\end{tabular} \\
\hline $\mathbf{n}$ & 18 & 20 & 24 & 25 & 27 & 28 & 29 \\
\hline$N$ & 29 & 32 & 40 & 42 & 46 & 48 & 50 \\
\hline $\mathrm{D} \mathrm{mm}$ & $4.8-5.1$ & 5.5 & $7.0-7.5$ & $12.3-14.1$ & 16.9 & 20.3 & 24.0 \\
\hline Abb. & $2 \mathrm{M}, \mathrm{L}$ & $2 \mathrm{~K}$ & $2 \mathrm{~J}$ & $2 \mathrm{G}, \mathrm{F}, \mathrm{E}, \mathrm{D}$ & $2 \mathrm{C}$ & $2 \mathrm{~B}$ & $2 \mathrm{~A}$ \\
\hline
\end{tabular}

Fig. 2. Czarnockia maroccana n. sp., paratype no. MB.-K.656., Clymenia genozone, El Atrous North. A. distal cross section (no. 1, with 2 lonsdaleioid dissepiments; detailed drawing of antiseptum and right antilateral septum including the 2 adhering minor septa and with 3 third order septa). B-G. calicular cross sections (no. 2, 5, 7-10, in B-E with normal dissepiments). H, I. median longitudinal sections near the calicular base (no. 11, 12, parallel to the plain of antiseptum and cardinal septum; antiseptum on the left). $\mathbf{J}$. cross section at the deepest calicular base (no. 13, with only 1 calicular chamber at the cardinal septum). K-M. subtabular cross sections (no. 16-18). $\times 2$ (A, with septal details $\times 9.25$ ), $\times 2.5(B), \times 3(\mathrm{C}), \times 3.5(\mathrm{D}-\mathrm{F})$, $\times 4(\mathrm{G}-\mathrm{I}), \times 6(\mathrm{~J})$, and $\times 8(\mathrm{~K}-\mathrm{M})$. Photos of corallum and sections see pl. 1: 7-10. Septal formulae. 


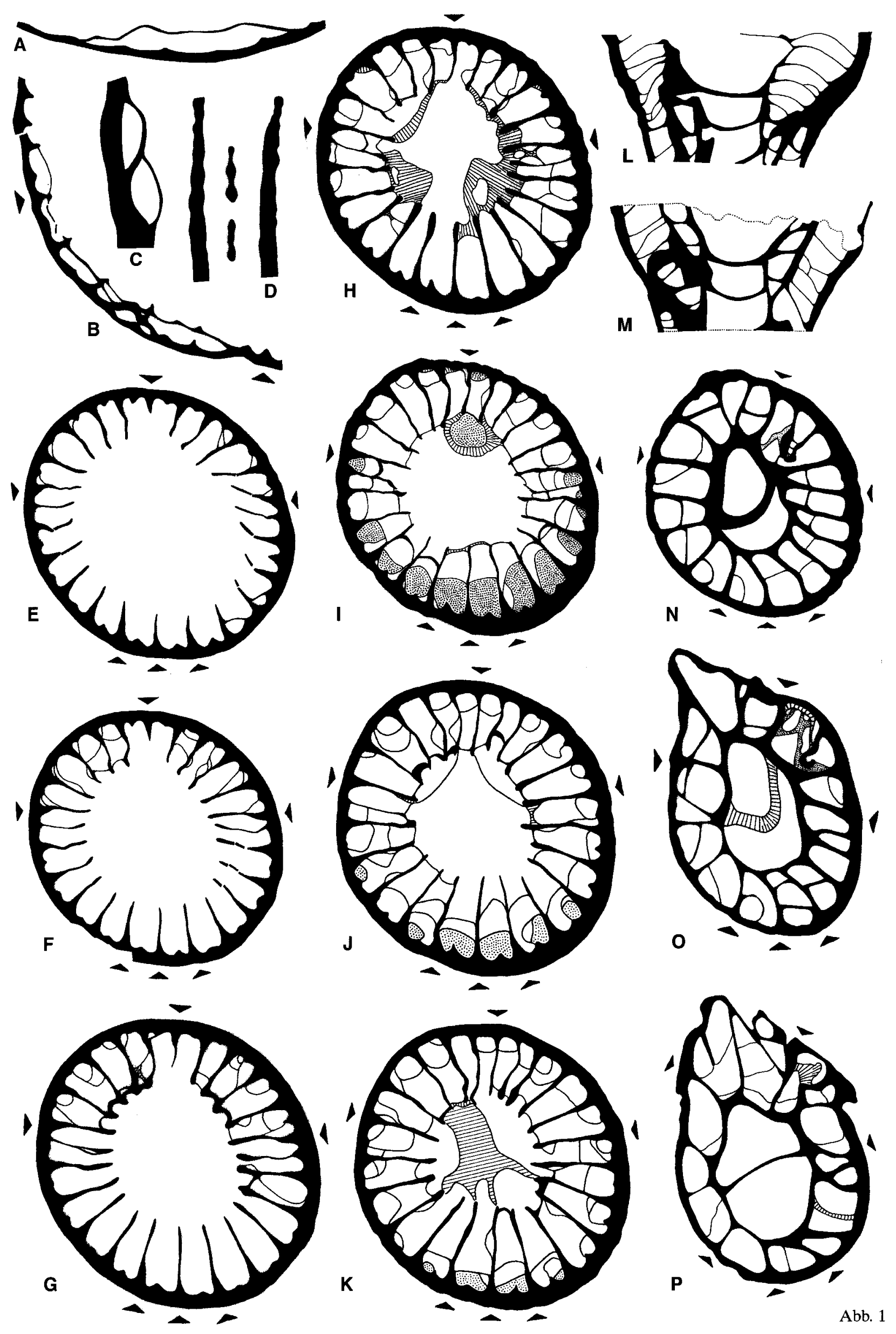



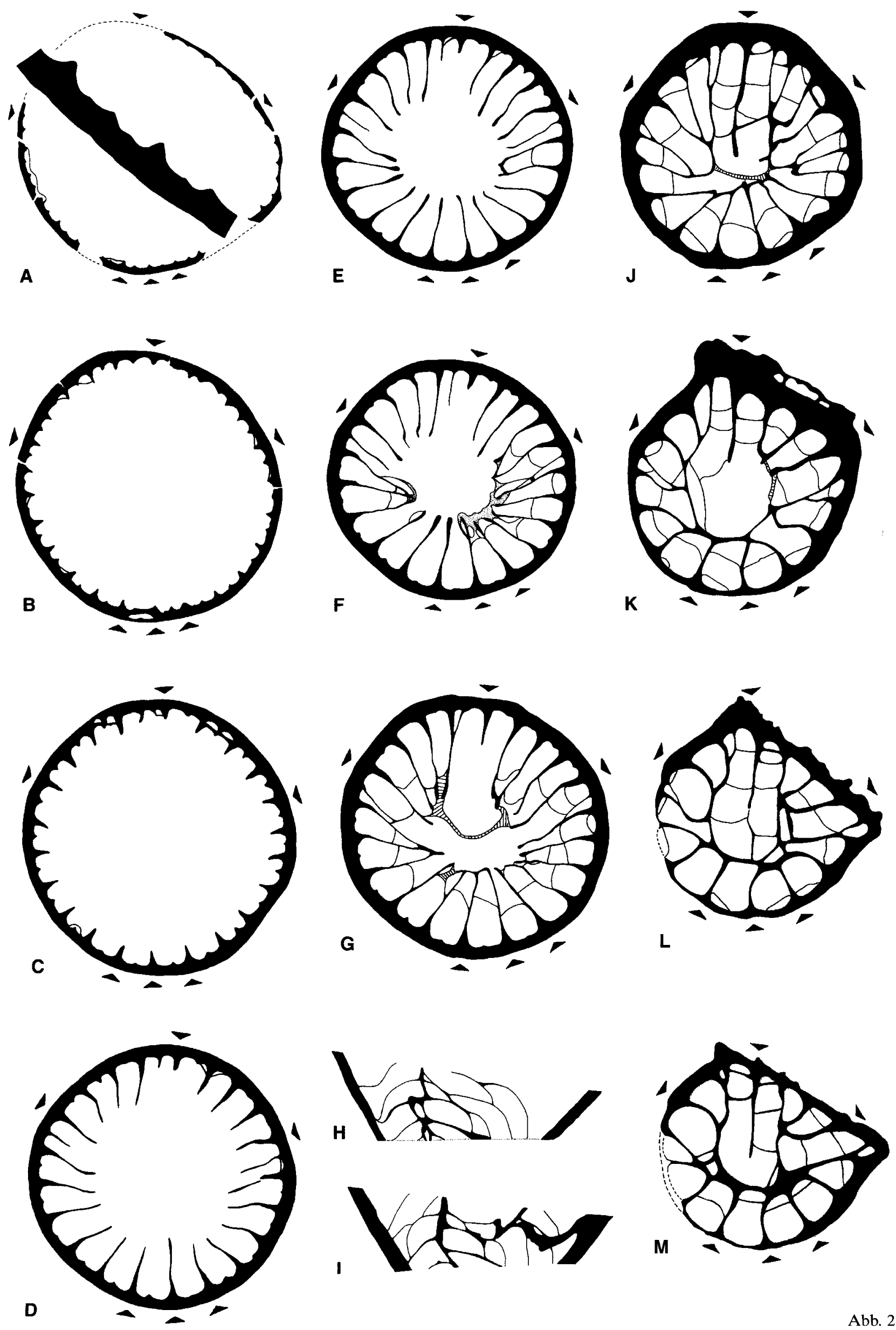

Abb. 2 

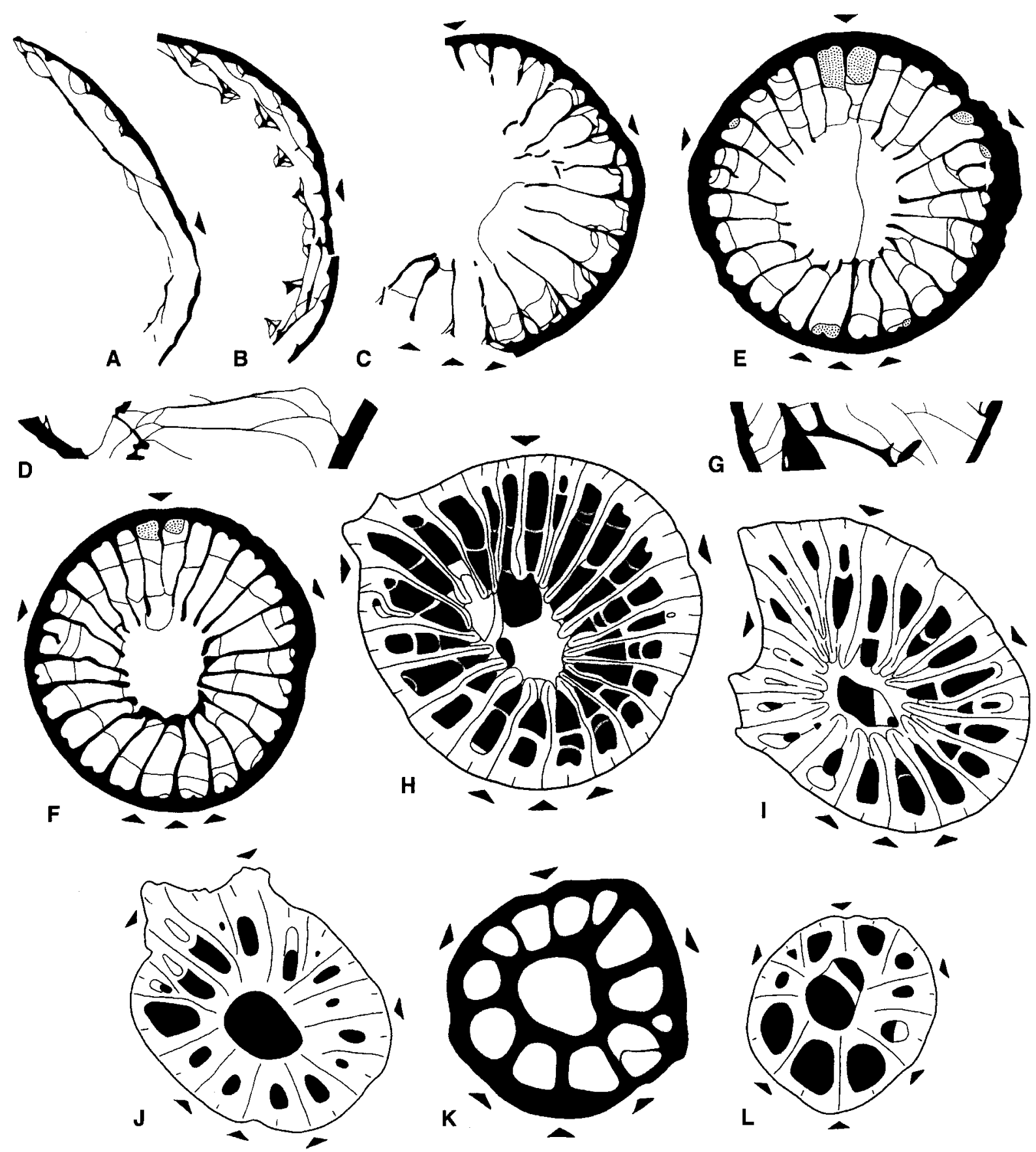

Abb. 3. Czarnockia maroccana n. sp., Paratypus Nr. MB.-K.657, Clymenia-Stufe, El Atrous Nord. A-C. caliculare Querschliffe (Nr. 1, 2, 5, distal mit einfachen und lonsdaleioiden Dissepimenten, $C$ im rejuvenescence-Niveau). D. medianer Längsschliff der Kelchbasis (Nr. 6, senkrecht zur Antiseptum/Cardinalseptum-Ebene, links 2 Dissepimentblasen). E, F. Querschliffe der Kelchbasis (Nr. 8, 9, periphere caliculare Anteile punktiert). G. subtabularer medianer Längsschliff (Nr. 10, parallel zur Antiseptum/Cardinalseptum-Ebene). H-L. subtabulare Querschliffe (Nr. 12, 15, 18-20). $\times 4(\mathrm{~A}-\mathrm{D}, \mathrm{G}), \times 4.5(\mathrm{E}, \mathrm{F}), \times 6(\mathrm{H}), \times$ $8(\mathrm{I}), \times 12(\mathrm{~J})$ und $\times 18(\mathrm{~K}, \mathrm{~L})$. Querschliff-Fotos siehe Tafel 3: 5-6. Septenformeln:

\begin{tabular}{|c|c|c|c|c|c|c|c|c|}
\hline \multirow[t]{2}{*}{ Nr. K.657 } & $2 \mid 2$ & \begin{tabular}{l|l}
2 & 3
\end{tabular} & \begin{tabular}{l|l}
3 & 4
\end{tabular} & \begin{tabular}{l|l}
4 & 4
\end{tabular} & \begin{tabular}{l|l}
5 & 4
\end{tabular} & \begin{tabular}{l|l}
5 & 5
\end{tabular} & \begin{tabular}{l|l}
5 & 5
\end{tabular} & \\
\hline & \begin{tabular}{l|l}
3 & 3 \\
\end{tabular} & \begin{tabular}{l|l}
3 & 4
\end{tabular} & \begin{tabular}{l|l}
4 & 4
\end{tabular} & \begin{tabular}{l|l}
6 & 6 \\
\end{tabular} & \begin{tabular}{l|l}
6 & 7
\end{tabular} & \begin{tabular}{l|l}
7 & 7
\end{tabular} & \begin{tabular}{l|l}
8 & 7
\end{tabular} & \begin{tabular}{l|l}
8 & 8
\end{tabular} \\
\hline $\mathrm{n}$ & 14 & 16 & 19 & 24 & 26 & 28 & 29 & 30 \\
\hline $\mathrm{N}$ & 20 & 24 & 31 & 40 & 44 & 48 & 50 & 52 \\
\hline $\mathrm{D} \mathrm{mm}$ & 1.7 & 2.1 & 3.3 & 6.2 & 7.6 & 8.8 & 10.4 & $11.5-13.0$ \\
\hline Abb. & $3 \mathrm{~L}$ & $3 \mathrm{~K}$ & $3 \mathrm{~J}$ & 3I & - & $3 \mathrm{H}$ & $3 F$ & $3 E$ \\
\hline
\end{tabular}

Fig. 3. Czarnockia maroccana n. sp., paratype no. MB.-K.657, Clymenia genozone, El Atrous North. A-C. calicular cross sections (no. 1, 2, 5, in the distal part with normal and lonsdaleioid dissepiments, $\mathrm{C}$ in a rejuvenescence level). D. median longitudinal sections at calicular base (no. 6, perpendicular to the plain of antiseptum and cardinal septum, on the left 2 dissepiments). E, F. cross sections of the calicular base (no. 8, 9, with stippled peripheral calicular spaces). G. subtabular median longitudinal section (no. 10, parallel to the plain of antiseptum and cardinal septum). $\mathbf{H}-\mathbf{L}$. subtabular cross sections (no. $12,15,18-20) . \times 4(\mathrm{~A}-\mathrm{D}, \mathrm{G}), \times 4.5(\mathrm{E}, \mathrm{F}), \times 6(\mathrm{H}), \times 8(\mathrm{I}), \times 12(\mathrm{~J})$, and $\times 18(\mathrm{~K}, \mathrm{~L})$. Photos of cross sections see pl. 3: 5-6. Septal formulae. 
A

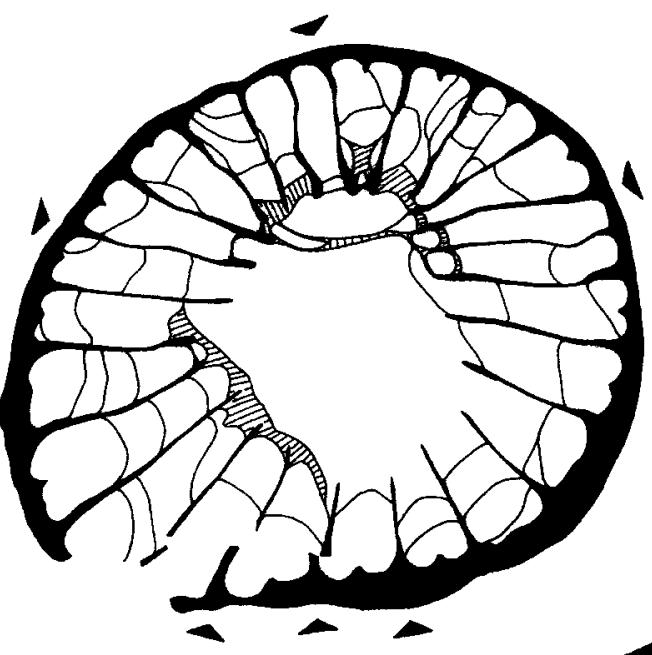

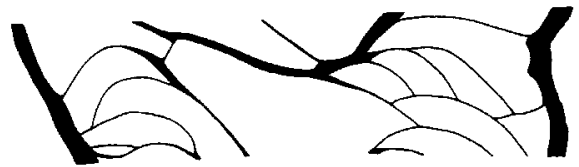

C

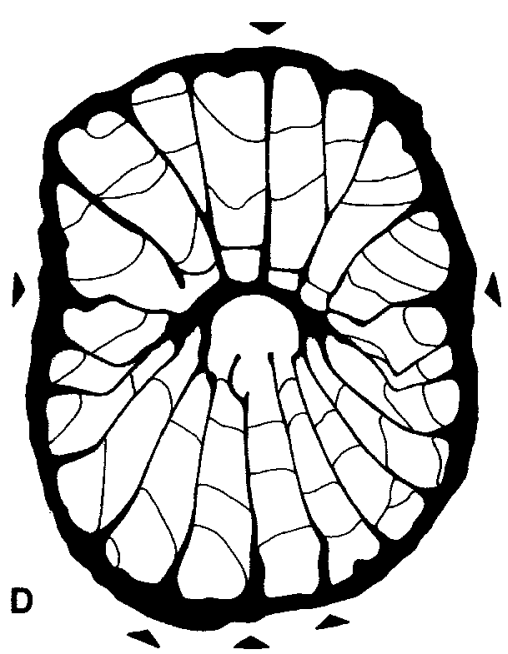

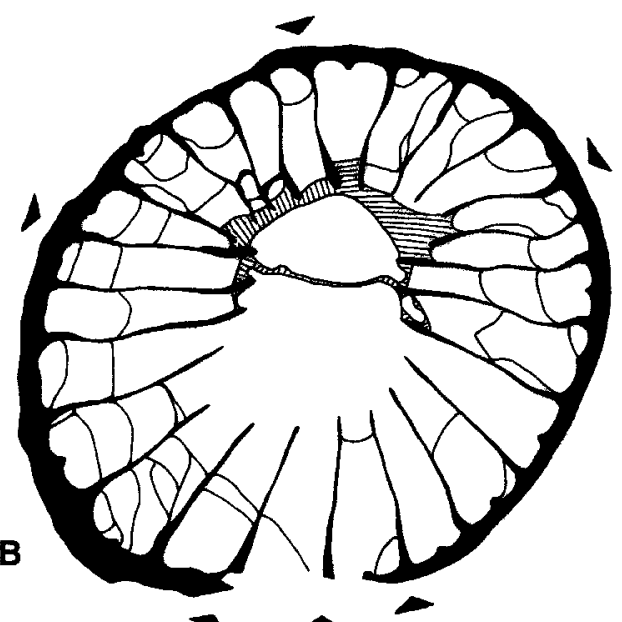

$\mathbf{E}$

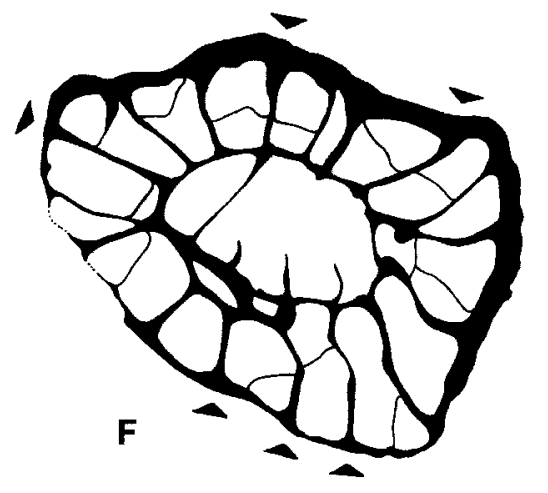

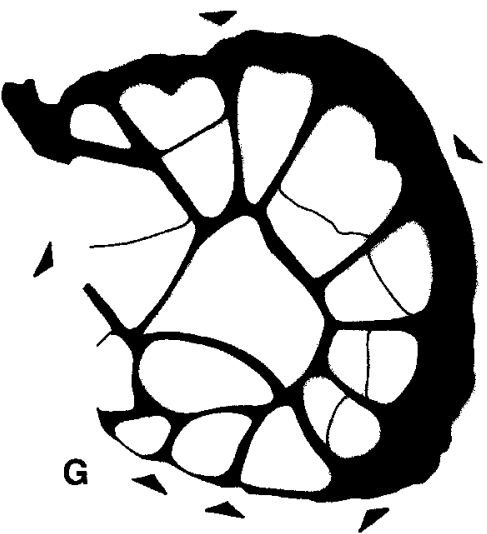

Abb. 4. Czarnockia maroccana n. sp., Paratypus Nr. MB.-K.658, Clymenia-Stufe, El Atrous Nord. A, B. Querschliffe der Kelchbasis (Nr. 3, 4, nur in den Antiquadranten peripher noch 7 und 3 caliculare Lumina). C. subtabularer medianer Längsschliff (Nr. 5, parallel zur Antiseptum/Cardinalseptum-Ebene, Antiseptum rechts). D-G. subtabulare Querschliffe (Nr. 8, 10-12). $\times 4(\mathrm{~A}-\mathrm{C}), \times 5(\mathrm{D}), \times 7(\mathrm{E}), \times 8(\mathrm{~F})$ und $\times 12(\mathrm{G})$. Querschliff-Foto siehe Tafel 3: 7. Septenformeln:

\begin{tabular}{lc|c} 
Nr. K.658 & 3 & 2 \\
\cline { 2 - 3 } & 4 & 4 \\
$\mathrm{n}$ & 17 \\
$\mathrm{~N}$ & 26 \\
$\mathrm{D} \mathrm{mm}$ & 2.6 \\
Abb. & $4 \mathrm{G}$
\end{tabular}

\begin{tabular}{c|c}
3 & 2 \\
\hline 5 & 5 \\
19 \\
31 \\
$4.3 \times 5.9$ \\
$4 \mathrm{~F}$
\end{tabular}

\begin{tabular}{c|c}
3 & 3 \\
\hline 5 & 5 \\
20 \\
32 \\
$5.0 \times 6.3$ \\
$4 \mathrm{E}$
\end{tabular}

\begin{tabular}{c|c}
4 & 3 \\
\hline 5 & 6 \\
22 \\
37 \\
$8.8 \times 11.3$ \\
$4 \mathrm{D}$
\end{tabular}

\begin{tabular}{c|c}
4 & 4 \\
\hline 6 & 7 \\
25 \\
42 \\
$10.4 \times 11.9$ \\
-
\end{tabular}

\begin{tabular}{c|c}
4 & 4 \\
\hline 7 & 7 \\
26 \\
44 \\
$3.8 \times 15.5$ \\
$4 \mathrm{~B}, \mathrm{~A}$
\end{tabular}

Fig. 4. Czarnockia maroccana n. sp., paratype no. MB.-K.658, Clymenia genozone, El Atrous North. A, B. cross sections of the calicular base (no. 3, 4, with still 7 and 3 calicular spaces only in the peripheral antiquadrants). C. subtabular median longitudinal section (no. 5, parallel to the plain of antiseptum and cardinal septum, antiseptum on the right). D-G. subtabular cross sections (no. 8, 10-12). $\times 4(\mathrm{~A}-\mathrm{C}), \times 5(\mathrm{D}), \times 7(\mathrm{E}), \times 8(\mathrm{~F})$, and $\times 12(\mathrm{G})$. Foto of cross section see pl. 3: 7. Septal formulae. 


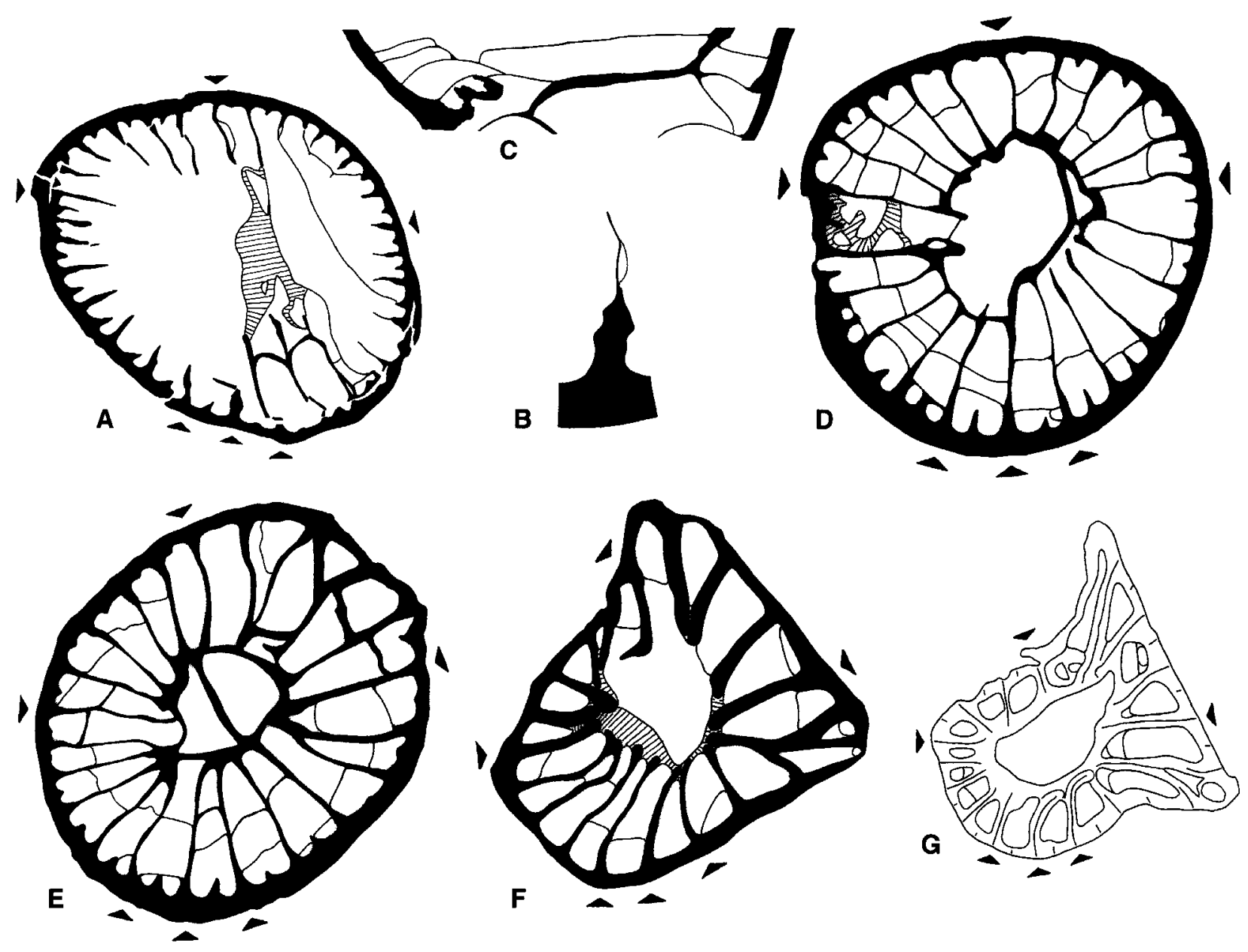

Abb. 5. Czarnockia maroccana n. sp., Paratypus Nr. MB.-K.659., Clymenia-Stufe, El Atrous Nord. A. distaler Kelchquerschliff (Nr. 1, links calicular, rechts subtabular nach amplexoider Verjüngung). B. Großseptum des rechten Antiquadranten von Querschliff Nr. 1, mit groben Trabekeln und Septalflankendissepiment (Periseptalblase). C. subtabularer medianer Längsschliff (Nr. 3, parallel zur Cardinalseptum/Antiseptum-Ebene). D. Querschliff der Kelchbasis (Nr. 5, nur im linken Antiquadranten peripher noch 5 caliculare Lumina). E-G. subtabulare Querschliffe (Nr. 7, 9, 10). $\times 3$ (A), $\times 15$ (B), $\times 4$ (C), $\times 5$ (D) und $\times 6$ (E-G). Polypar- und Querschliff-Foto siehe Tafel 2: 7-8. Septenformeln:

\begin{tabular}{|c|c|c|c|c|c|c|}
\hline \multirow[t]{2}{*}{ Nr. K.659 } & \begin{tabular}{l|l}
3 & 4 \\
\end{tabular} & \begin{tabular}{l|l}
4 & 4
\end{tabular} & \begin{tabular}{l|l}
5 & 5 \\
\end{tabular} & \begin{tabular}{l|l}
5 & 5 \\
\end{tabular} & & \begin{tabular}{l|l}
6 & 6
\end{tabular} \\
\hline & \begin{tabular}{l|l}
4 & 5
\end{tabular} & \begin{tabular}{l|l}
5 & 5
\end{tabular} & \begin{tabular}{l|l}
5 & 6
\end{tabular} & \begin{tabular}{l|l}
6 & 6
\end{tabular} & \begin{tabular}{l|l}
77 & 6
\end{tabular} & \begin{tabular}{l|l}
8 & 8
\end{tabular} \\
\hline $\mathrm{n}$ & 20 & 22 & 25 & 26 & 27 & 32 \\
\hline $\mathrm{N}$ & 32 & 36 & 42 & $44-46$ & 47 & 57 \\
\hline $\mathrm{D} \mathrm{mm}$ & 4.7 & 8.4 & $9.6 \times 7.6$ & $8.1 \times 9.8-10.2 \times 9.9$ & 11.0 & $14.3 \times 18.6$ \\
\hline Abb. & $5 \mathrm{G}$ & $5 \mathrm{~F}$ & - & $5 \mathrm{E}$ & 5D & $5 \mathrm{~A}$ \\
\hline
\end{tabular}

Fig. 5. Czarnockia maroccana n. sp., paratype no. MB.-K.659., Clymenia genozone, El Atrous North. A. cross section of the distal calice (no. 1, calicular on the left, subtabular on the right due to an amplexoid rejuvenescence). B. major septum from the right antiquadrant of cross section no. 1 (with coarse trabeculae and a periseptal dissepiment). C. subtabular median longitudinal section (no. 3, parallel to the plain of antiseptum and cardinal septum). D. cross section of the calicular base (no. 5, with only 5 peripheral caliculae luminae in the left antiquadrant). E-G. subtabular cross sections (no. 7, 9, 10). $\times 3$ (A), $\times 15$ (B), $\times 4(C), \times 5(D)$, and $\times 6(E-G)$. Photos of corallum and cross section see pl. 2: 7-8. Septal formulae.

Kelchrand zeigt, dass hier - wie bei Neaxon regulus (Richter, 1848) und im Unterschied zu Hyposepten (Weyer 1997) - noch kein Platz für einen zusätzlichen Septensatz (neben Proto-/Metasepten und Katasepten) gegeben ist.

Die trabekuläre Septenmikrostruktur wurde vereinzelt bei lokaler Steinkernerhaltung direkt in Form grober Septalrandspinae im distalen Kelch festgestellt. Dort ist dieser Bauplan auch im tangentialen Längsschliff sowie in Querschliff- details sichtbar (Abb. 1D, 5B). Eine multitrabekuläre Septenstirnzone unmittelbar am Kelchrand war nicht nachzuweisen.

Ein typischer Aulos (komplette Ringwand) als Neaxon-Erbgut ist auf den Jugendstadien (bei $\mathrm{D}$ 1.7-3.4 mm, maximal $7 \mathrm{~mm}$ ) anzutreffen; er variiert von klein (Abb. 3J-L, 7K) bis groß (Abb. 1N-P). Seine meist stereothecate Struktur (Tafel 2: 6) kann auch phyllothecat sein (Tafel 1: 6). Schon früh wird sein Bau unvollständig und 

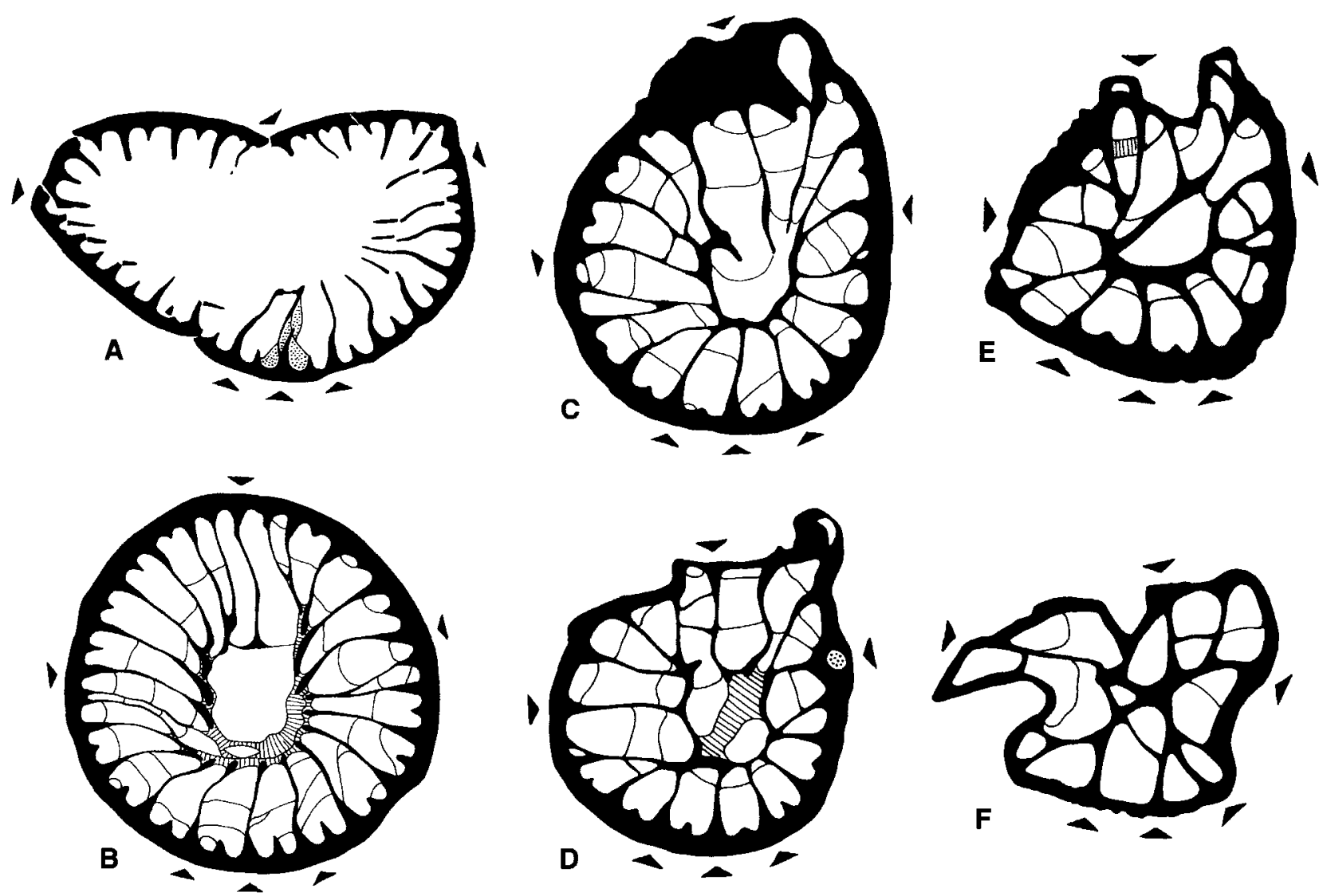

Abb, 6. Czarnockia maroccana n. sp., Paratypus Nr. MB.-K.660, Clymenia-Stufe, El Atrous Nord. A-F. Querschliffserie (Nr. 4, 6, 10-13), calicular (A, mit Dissepimenten am Antiseptum), Kelchbasis (B, mit calicularer Detritusfüllung in den peripheren Interseptalräumen der Hauptquadranten) und subtabular $(\mathrm{C}-\mathrm{F}) \times 3(\mathrm{~A}), \times 4(\mathrm{~B}), \times 6(\mathrm{C}, \mathrm{D}), \times 8(\mathrm{E})$ und $\times 10(\mathrm{~F})$. Querschliff-Fotos siehe Tafel 3:1-4. In D ist am rechten Lateralseptum in der Wand ein Fremdkörper eingeschlossen. Septenformeln:

Nr. K.660

n

$\mathrm{N}$

$\mathrm{D}$ mm

Abb.

\begin{tabular}{c|c}
2 & 2 \\
\hline 3 & 3 \\
14 \\
20 \\
3.0 \\
$6 \mathrm{~F}$
\end{tabular}

\begin{tabular}{c|c}
3 & 2 \\
\hline 3 & 4 \\
16 \\
25 \\
4.8 \\
$6 \mathrm{E}$
\end{tabular}

\begin{tabular}{|c|c|}
\hline 413 & 3 \\
\hline \begin{tabular}{l|l}
4 \\
\end{tabular} & 4 \\
\hline 19 & \\
\hline 30 & \\
\hline 6.1 & \\
\hline $6 \mathrm{D}$ & \\
\hline
\end{tabular}

\begin{tabular}{l|l}
5 & 4 \\
\hline 6 & 6 \\
25 \\
42 \\
$11.3-11.8$
\end{tabular}

$6 \mathrm{~B}$

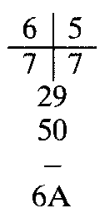

$6 \mathrm{~A}$

Fig. 6. Czarnockia maroccana n. sp., paratype no. MB.-K.660, Clymenia genozone, El Atrous North. A-F. Series of cross sections (no. 4,6,10-13), calicular (A, with dissepiments near antiseptum), base of calice (B, with calicular detritus in the peripheral interseptal spaces of the cardinal quadrants), and subtabular (C-F). $\times 3(\mathrm{~A}), \times 4(\mathrm{~B}), \times 6(\mathrm{C}, \mathrm{D}), \times 8(\mathrm{E})$, and $\times$ 10 (F). Photos of cross sections see pl. 3: 1-4. The wall of D contains a foreign particle near the right lateral septum. Septal formulae.

Abb. 7. Czarnockia maroccana n. sp., Paratypus Nr. MB.-K.661, Clymenia-Stufe, Ouidane Chebbi Südost. A-L. Querschliffserie (Nr. 2-8,10-12,14,15), calicular (A-F, in E mit 6 Dissepimentblasen), Kelchbasis (G, in den Antiquadranten peripher noch 6 caliculare Lumina) und subtabular $(\mathrm{H}-\mathrm{L}) . \times 4(\mathrm{~A}-\mathrm{C}), \times 4.5(\mathrm{D}-\mathrm{F}), \times 6(\mathrm{G}), \times 7(\mathrm{H}), \times 9(\mathrm{I}), \times 10(\mathrm{~J})$ und $\times 12$ $(\mathrm{K}, \mathrm{L})$. Polypar- und Querschliff-Fotos siehe Tafel 2: 1-6. Archaeotheca durch Winderosion stark korrodiert. Septenformeln:

\begin{tabular}{|c|c|c|c|c|}
\hline \multirow[t]{2}{*}{ Nr. K.661 } & \begin{tabular}{l|l}
2 & 2 \\
\end{tabular} & \begin{tabular}{l|l}
3 & 3 \\
\end{tabular} & \begin{tabular}{l|l}
3 & 3
\end{tabular} & \begin{tabular}{l|l}
4 & 4 \\
\end{tabular} \\
\hline & \begin{tabular}{l|l}
4 & 4
\end{tabular} & \begin{tabular}{l|l}
4 & 4
\end{tabular} & \begin{tabular}{l|l}
4 & 5
\end{tabular} & \begin{tabular}{l|l}
5 & 5
\end{tabular} \\
\hline $\mathrm{n}$ & 16 & 18 & 19 & 22 \\
\hline $\mathrm{N}$ & 24 & 28 & $30-33$ & 36 \\
\hline $\mathrm{D} \mathrm{mm}$ & $2.8-3.2$ & 3.8 & $4.2-5.2$ & 6.9 \\
\hline Abb. & 7L,K & - & 7J,I & $7 \mathrm{H}$ \\
\hline
\end{tabular}

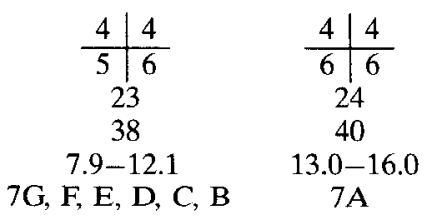

Fig. 7. Czarnockia maroccana n. sp., paratype no. MB.-K.661, Clymenia genozone, Ouidane Chebbi Southeast. A-L. Series of cross sections (no. 2-8,10-12,14,15), calicular $(\mathrm{A}-\mathrm{F}$, with 6 dissepiments in $\mathrm{E}$ ), base of calice (G, with 6 peripheral calicular spaces in the antiquadrants), and subtabular $(\mathrm{H}-\mathrm{L}) \times 4(\mathrm{~A}-\mathrm{C}), \times 4.5(\mathrm{D}-\mathrm{F}), \times 6(\mathrm{G}), \times 7(\mathrm{H}), \times 9(\mathrm{I}), \times 10(\mathrm{~J})$, and $\times$ $12(\mathrm{~K}, \mathrm{~L})$. Photos of corallum and cross sections see pl. 2: 1-6. The archaeotheca is strongly corroded by wind-erosion. Septal formulae. 

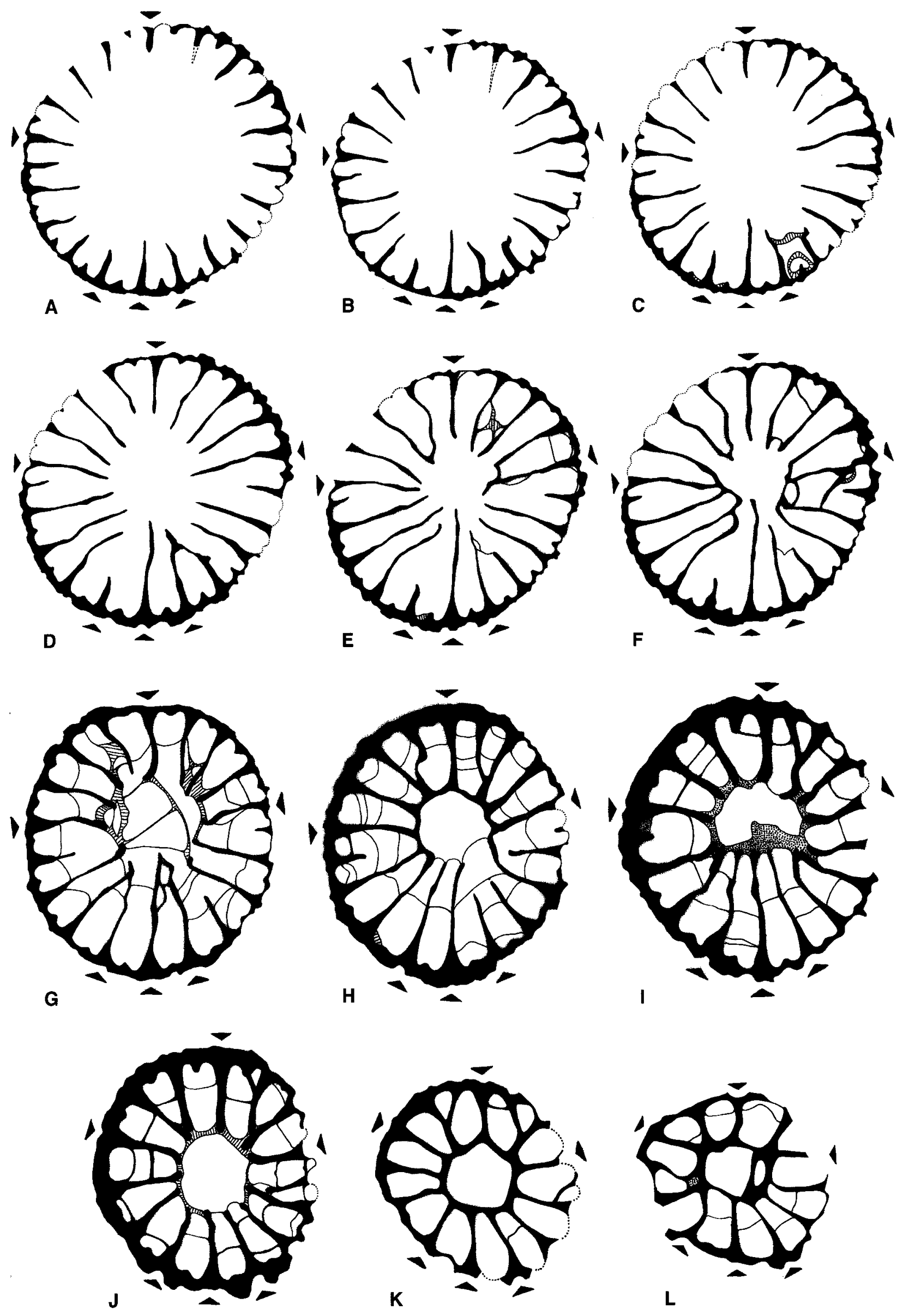
örtlich unterbrochen wie bei den Petraia-Vorfahren (Abb. $2 \mathrm{~K}-\mathrm{M}, 4 \mathrm{E}, \mathrm{F}$, um $5 \mathrm{~mm} \mathrm{D}$, maximal bis $11 \mathrm{~mm} \mathrm{D)}$. Adult verschwindet der Aulos vollständig - die Großsepten enden axial frei (Abb. 1G-K, 3E) und werden diskontinuierlich (je nach Anschnitt der Böden) lediglich durch Tabulae und Tabellae verbunden, die genau an der Stelle der vorherigen Aulos-Ringwand eine everte Zone im Tabularium bilden. Nur ausnahmsweise findet sich noch einmal vorübergehend eine subzentrale Septenverbindung (Abb. 7F).

Das adulte Tabularium ist insgesamt aufgewölbt und besteht vom Zentrum zur Peripherie hin aus drei Zonen (axiale Depression, everter Bereich, Abfall zur Wand - Abb. $2 \mathrm{H}, \mathrm{I}, 4 \mathrm{C}$ ), die von relativ wenigen weitspannigen Tabellae gebildet werden. Bei noch späteren Stadien und speziell in rejuvenescence-Fällen tritt eine Verflachung ein; bei nur geringer Gesamtaufwölbung verringert sich die Anzahl der Tabellae, und stattdessen treten einige durchgehende Tabulae auf (Abb. 3D, 5C). Juvenil (Abb. 1L, M) dominieren konkave Aulos-Böden und außerhalb der Ringwand einfache, zentrifugal abtauchende Tabulae.

Viele Querschliffbilder zeigen den klaren Ansatz eines leicht biformen Tabularium-Bauplans. Bodenschnitte in den Interseptalräumen verlaufen nicht tangential parallel zur Wand, sondern schräg (wandnah in der antiseptalen, wandfern in der cardinalen Hälfte eines Lumens -
Abb. 4D). An der Kelchbasis entspricht dem ein letztes Verbleiben winziger calicularer Anteile in der cardinalen Lumina-Hälfte (Abb. $1 \mathrm{~J}$, K, Tafel 1: 3). Besonders auffällig ist das typische Erscheinungsbild am Antiseptum mit einer Umorientierung der schiefen Tabulaeschnitte (Abb. 2J, 4B, 6C), was bekanntlich die leichtere Identifizierung der Protosepten ermöglicht.

Eine echte Fossula ist gut ausgebildet und an der Kelchbasis sofort erkennbar (Detritusfüllung reicht am Cardinalseptum tiefer hinab als in benachbarten Interseptallumina). Nur scheinbar spricht dagegen die noch tiefere Präsenz calicularer Bereiche in den Antiquadranten (Abb. 1J-K, 4A-B, 5D, 7G), aber dort erfolgte die Querschliff-Präparation nicht konsequent parallel zum Kelchoberrand, sondern kompromissbereit mehr an der Anwachsstreifung orientiert, sodass zwangsläufig (nach Weyer 1973: Abb. 4, 5) cardinal schon ein jeweils tieferes Kelchniveau vorliegt als antiseptal. Das ist mehrfach auch der Grund, weshalb das Cardinalseptum calicular manchmal in den abgebildeten Schliffserien (Abb. 4-6) kaum verkürzt vorliegt (aber an nicht abgebildeten Querschliffen oder an den Reststücken doch typisch verkürzt festgestellt werden konnte).

Einfache normale Dissepimente erscheinen erst im Alter, in der Regel also im unteren und mittleren Kelch (Abb. 1E, F, 2B-E, 3D, 7E, Tafel 1: 2); nur vereinzelt treten sie auch schon in den höchsten subtabularen Bereichen auf

Legenden zu den Tafeln 1 bis 3

Tafel 1. Czarnockia maroccana n. sp., Clymenia-Stufe, El Atrous Nord. 1-6. Holotypus Nr. MB.-K.655. 1. Polypar in Seitenansicht, $\times$ 2. 2-4. caliculare Querschliffe $(\mathrm{Nr} .10,12,14), \times 3.5(2)$ und $\times 4(3,4) .5$. medianer Längsschliff $(\mathrm{Nr} .17), \times 5.6$. subtabularer Querschliff (Nr. 21), $\times$ 10. (Schliffzeichnungen siehe Abb. 1A-P). 7-10. Paratypus Nr. MB.-K.656. 7. Polypar in Seitenansicht, $\times 3.8-10$. Querschliffe (Nr. 10,15,17), $\times 4, \times 8$ und $\times 10$. (Schliffzeichnungen siehe Abb. 2A-M).

Plate 1. Czarnockia maroccana n. sp., Clymenia genozone, El Atrous North. 1-6. Holotype no. MB.-K.655. 1. lateral view of corallum, $\times 2$. 2-4. calicular cross sections (no. $10,12,14), \times 3.5(2)$, and $\times 4(3,4)$. 5. median longitudinal section (no. 17), $\times$ 5. 6. subtabular cross section (no. 21) $\times 10$. (drawings of sections see Fig. 1A-P). 7-10. Paratype no. MB.-K.656. 7. lateral view of corallum, $\times 3.8-10$. Cross sections (no. 10,15,17), $\times 4, \times 8$, and $\times 10$. (drawings of sections see Fig. $2 \mathrm{~A}-\mathrm{M}$ ).

Tafel 2. Czarnockia maroccana n. sp., Clymenia-Stufe. 1-6. Paratypus Nr. MB.-K.661. Ouidane Chebbi Südost. 1. Polypar in Seitenansicht, $\times 2$. 2-6. Querschliffe (Nr. 5, 7, 11, 12, 14), $\times 5(2,3), \times 12(4), \times 13(5)$ und $\times 16$ (6). (Schliffzeichnungen siehe Abb. 7A-L), 7-8. Paratypus Nr. MB. K.659. El Atrous Nord. 7. Polypar in Seitenansicht, $\times 3.8$. Querschliff $($ Nr. 6), $\times$ 6. (Schliffzeichnungen siehe Abb. 5A-G).

Plate 2. Czarnockia maroccana n. sp., Clymenia genozone. 1-6. Paratype no. MB.-K.661. Ouidane Chebbi Southeast. 1. lateral view of corallum, $\times 2.2-6$. Cross sections (no. 5, 7, 11, 12,14), $\times 5(2,3), \times 12(4), \times 13(5)$, and $\times 16(6)$. (drawings of sections see Fig. 7A-L). 7-8. Paratype no. MB.-K.659. El Atrous North. 7. Lateral view of corallum, $\times 3$. 8. Cross section (no. 6), $\times 6$. (drawings of sections see Fig. $5 \mathrm{~A}-\mathrm{G}$ ).

Tafel 3. Czarnockia maroccana n. sp., Clymenia-Stufe, El Atrous Nord. 1-4. Paratypus Nr. MB.-K.660. Querschliffe (Nr. 5, 7, $8,11), \times 4(1), \times 5.2(2), \times 6(3)$ und $\times 8$ (4). (Schliffzeichnungen siehe Abb. 6A-F). 5, 6. Paratypus Nr. MB.-K.657. Querschliffe $(\mathrm{Nr} .9,14), \times 5$ und $\times 7$. (Schliffzeichnungen siehe Abb. 3A-L). 7. Paratypus Nr. MB.-K.658. Querschliff $(\mathrm{Nr} .7), \times 5$. (Schliffzeichnungen siehe Abb. $4 \mathrm{~A}-\mathrm{G}$ ).

Plate 3. Czarnockia maroccana n. sp., Clymenia genozone, El Atrous North. 1-4. Paratype no. MB.-K.660. cross sections (no. $5,7,8,11), \times 4(1), \times 5.2(2), \times 6(3)$, and $\times 8(4)$. (drawings of sections see Fig. 6A-F). 5, 6. Paratype no. MB.-K.657. cross sections (no. 9, 14), $\times 5$ and $\times 7$. (drawings of sections see Fig. 3A-L). 7. Paratype no. MB.-K.658, cross section (no. 7), $\times 5$. (drawings of sections see Fig. $4 \mathrm{~A}-\mathrm{G}$ ). 


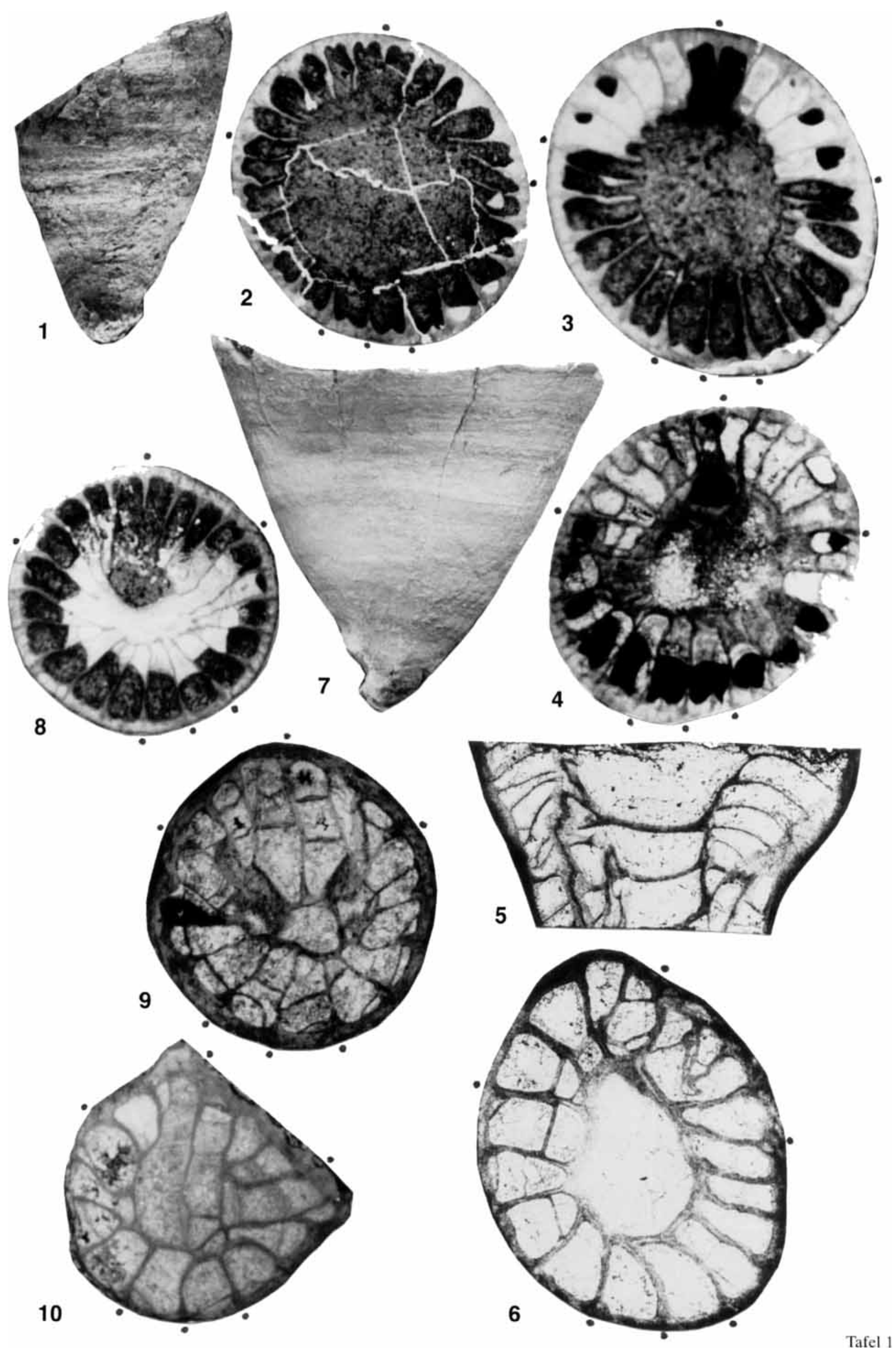



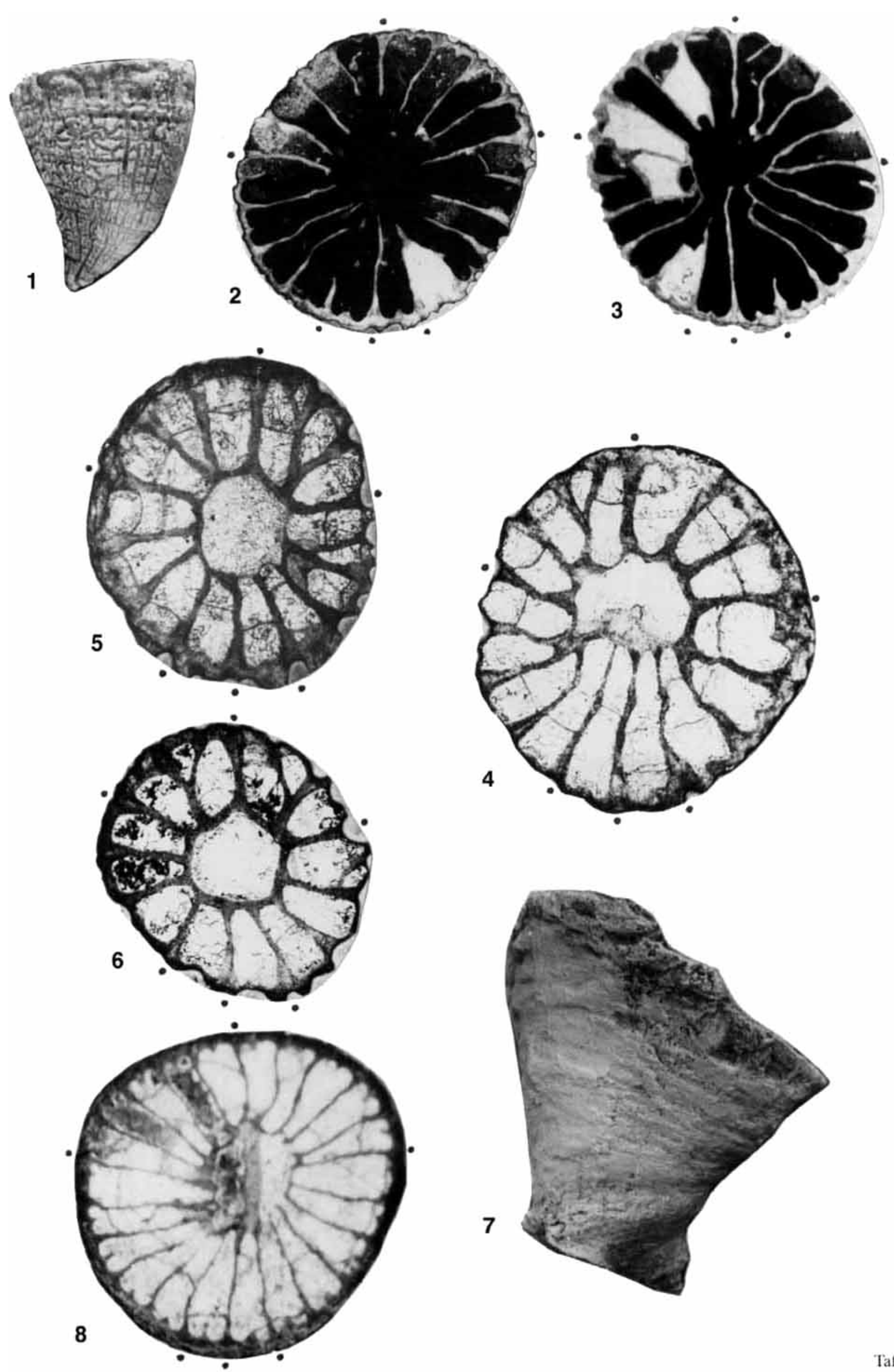

Tafel 2 


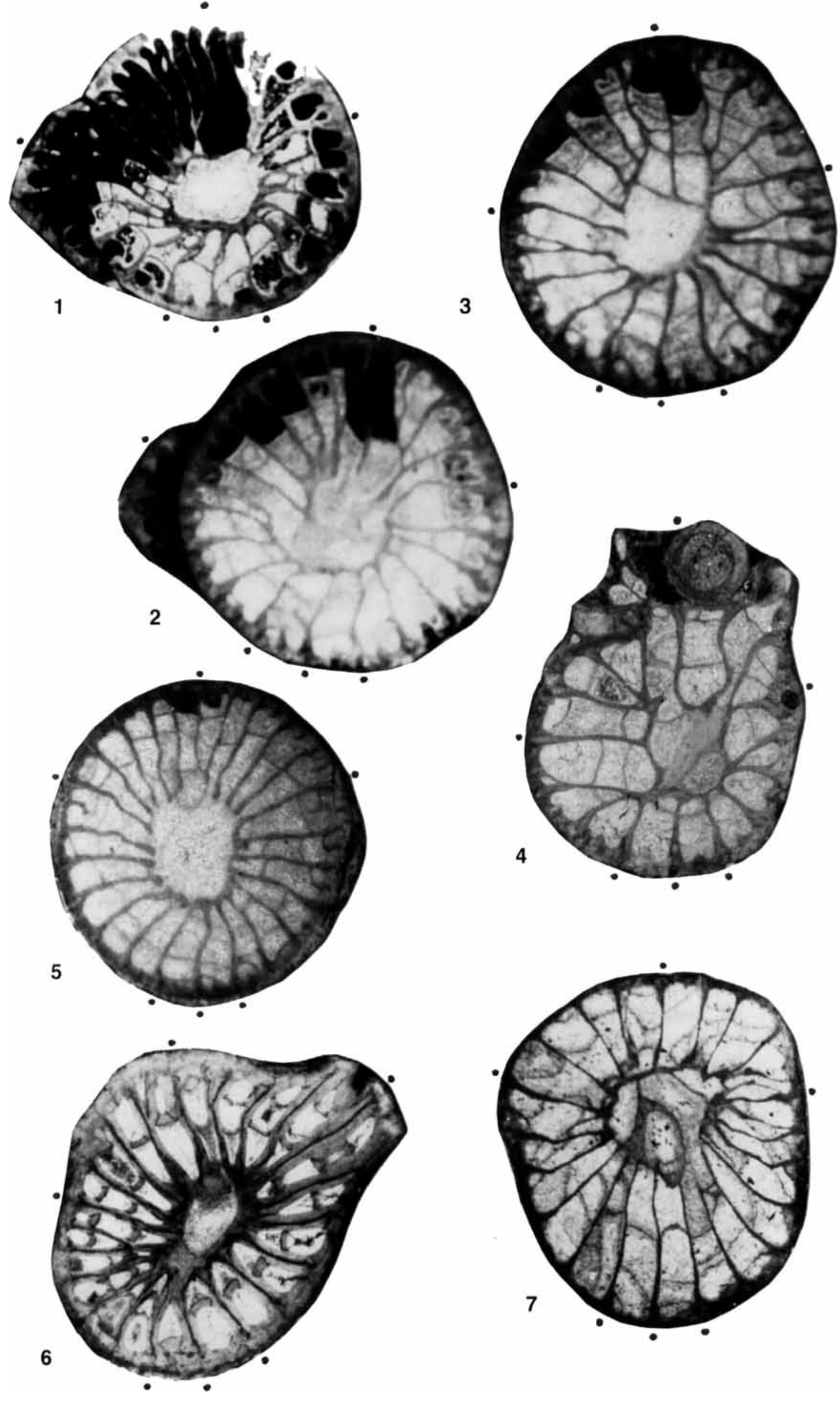

Tafel 3 
(Abb. 4A, B). Nie aber bilden sie eine an der Peripherie durchgehend ausgebildete Zone. Neben gewöhnlich einreihiger Anlage kommt selten auch ein mehrreihiger Bauplan vor (Abb.3B). Häufig gehen die tangential orientierten wandnahen Dissepimente kontinuierlich in radial ausgerichtete Septalflankendissepimente über (Abb. 1F, 3C), die zusätzlich auch an der inneren Großseptenhälfte vorkommen (Bereich der aus dem Aulos hervorgegangenen everten TabellaeZone - Abb. 1G, K, 7G). Die subtabulare Unterscheidung von Dissepiment und wandnahem Tabula-Schnitt ist ziemlich eindeutig (konkav oder konvex zum Polyparzentrum orientiert Abb. 1I). Theoretisch könnten konkave Schnitte auch Böden darstellen, die nach generellem zentrifugalen Abtauchen kurz vor Erreichen der Wand sich wieder etwas aufwärts biegen; nach Aussage der Längsschliffe ist das aber nur äuBerst selten der Fall (Abb. 4C). Im oberen Kelch können lonsdaleioide Dissepimente hinzukommen (Abb. 1A, B, 2A, 3A, B). Im Bereich des Anheftungstalons gibt es verständlicherweise einige Wachstumsanomalien und Talon-Dissepimente (Abb. 1N-P, 2K, 6C-E).

Bemerkungen: Die Variationsbreite der sieben verfügbaren Polypare ist nach Aussage ihrer ausführlichen Schliffserien relativ erheblich, sodass vorübergehend Zweifel bestanden, sie alle nur einer Art zuzuordnen. Speziell beim von einem anderen Fundort stammenden Paratypus der Abb. 7 mit seinem leicht verlängerten Antiseptum und den ontogenetisch früh prominenten Kleinsepten war zunächst an ein selbständiges Taxon gedacht, doch findet sich derselbe Kleinsepten-Bauplan auch bei einem Exemplar aus der Population vom Fundort El Atrous Nord wieder (Abb. 6). Andere Kriterien erwiesen sich wegen ihrer mosaikartigen Verteilung ebenso untauglich für spezifische Abtrennungen: die geringfügig höhere Septen-Anzahl und die stärkere juvenile Skelettverdickung in Abb. 3, 5, die Präsenz von Meiosepten (Abb. 2, 4, 7), das lediglich schwach verkürzte Cardinalseptum (Abb. 4, 5, 7), der juvenil komplette Aulos in Abb. 3, 4, 7, die schwankende Aulos-Größe (minimal in Abb. 3, 6, 7) oder die gut ausgebildete Zone everter Tabellae im Bereich der vorherigen jugendlichen Aulos-Ringwand (Abb. 1-4, 7).

Beziehungen: Die Typusart Czarnockia obliqua Różkowska, 1969 als derzeit einziger weiterer Vertreter der Gattung unterscheidet sich durch ihr komplexeres Tabularium (mit reichlich Tabellae) und das vermutliche Fehlen lonsda- leioider Dissepimente im distalen Kelch (trotz der beachtlichen Polypargröße). Im adulten Querschliffbild stimmt speziell der Paratypus von Abb. 3 (mit etwas höherer Septen-Anzahl) weitgehend überein; Jugendstadien sind von der polnischen Form noch unbekannt.

Der Paratypus von Czarnockia obliqua Róźkowska, 1969 - nach meiner Präparation (Weyer 2000: Tafel 2-3) vermutlich ein eigenes, leider noch ungenügend bekanntes Taxon - ähnelt einem Paratypus der neuen marokkanischen Art (Abb. 7), entfernt sich aber deutlich durch seine proportional wesentlich dickeren Septen und schmaleren Interseptalräume in tieferen Kelchstadien.

Czarnockia simplex Różkowska, 1969 (Wocklumeria-Stufe, doVI, Heiligkreuz-Gebirge, Polen) ist meines Erachtens ein auf mangelhaftes und unzureichend präpariertes Material begründetes nomen dubium - vorerst generisch und spezifisch unbestimmbar (Weyer 2000: 81) - und entfällt für einen Vergleich.

Vorkommen: Tafilalt (El Atrous Nord, Ouidane Chebbi Südost), Antiatlas, Marokko; OberFamennium (mittlere/obere Clymenia-Stufe); Kollektion D. Weyer 1995 (7 Polypare).

\section{Danksagung}

Mein herzlicher Dank gilt Dr. Mohammed Dahmani (Rabat, Ministère de l'Énergie et des Mines) für die freundliche Genehmigung geologisch-paläontologischer Feldarbeiten und Aufsammlungen, Dr. Dieter Korn und Dr. Christian Klug (beide Tübingen) für die Geländeführung zu den marokkanischen Fundorten ihrer eigenen Ammonoidea-Studien, sowie Prof Dr. Jürgen Kullmann (Tübingen) und Dr. Stefan Schröder (Köln) für kritische Review-Notizen.

\section{Literatur}

Becker, R. T. 1993. Stratigraphische Gliederung und Ammonoideen-Faunen im Nehdenium (Oberdevon II) von Europa und Nord-Afrika. - Courier Forschungsinstitut Senckenberg 155: 1-405.

Belka, Z., Klug, C., Kaufmann, B., Korn, D., Döring, S., Feist, R. \& Wendt, J. 1999. Devonian conodont and ammonoid succession of the eastern Tafilalt (Ouidane Chebbi section), Anti-Atlas, Morocco. - Acta Geologica Polonica $49(1)$ : 1-23.

Berkowski, B. 2001. Famennian colonial Rugosa from southern Poland: recovery and extinction. - Bulletin of the Tohoku University Museum, 1 (Proceedings of the 8th International Symposium on Fossil Cnidaria and Porifera September 12-16, 1999 Sendai, Japan): 285-290; Sendai.

Dobrolyubova, T. A. \& Kabakovich, N. V. 1966. Chetyrekhluchevye korally nizhnego karbona Kuznetskoy kotloviny. [Tetracorals from the Lower Carboniferous of the Kuznetsk basin]. In Dobrolyubova, T. A., Kabakovich, N. V. 
\& Sayutina, T. A. (eds): Korally nizhnego karbona Kuznetskoy kotloviny. - Trudy Paleontologicheskogo Instituta, Akademiya Nauk SSSR, 111: 5-198, 217-223, 225-260; Moskva.

Dybowski, W. 1873-1874. Monographie der Zoantharia sclerodermata rugosa aus der Silurformation Estlands, Nord-Livlands und der Insel Gotland, nebst einer Synopsis aller palaeozoischen Gattungen dieser Abtheilung und einer Synonymik der dazu gehörigen, bereits bekannten Arten. - Archiv für die Naturkunde Liv-, Ehst- und Kurlands, Serie 1, 5 (3,4): 257-532; Dorpat [1873: 257-414, pls. 1-2; 1874: 415-532, pls. 3-5].

Frech, F. 1885. Die Korallenfauna des Oberdevons in Deutschland. - Zeitschrift der Deutschen Geologischen Gesellschaft 37 (1): 21-130.

Gorskiy, I. I. 1938. Kamennougol'nye korally Novoy Zemli [Carboniferous corals from Novaya Zemlya]. - Trudy, Vsesoyuznyy Arkticheskiy Institut 93 (Paleontologiya Sovetskoy Arktiki, II): 1-221; Leningrad.

Gorskiy, I. I., Degtyarev, D. D., Kachanov, E. I., Rakshin, P. P. \& Simakova, M. A. 1975. Tetracoralla (Chetyrekhluchevye korally) (Rugosa). - Trudy, Vsesoyuznyy Neftyanoy Nauchno-Issledovatel'skiy Geologorazvedochnyy Institut (VNIGRI), 383 (Stepanov, D. L. et al., eds: Paleontologicheskiy atlas kamennougol'nykh otlozheniy Urala): 1-360, 2 figs., 2 tabs., 100 pls. (73-89, 224-226, pls. 21-28); Leningrad.

Guo Sheng-zhe 1990. Frasnian-Famennian extinction and late Devonian rugose corals from Great Xing'an ranges, NE China. - Acta Palaeontologica Sinica, 29 (4): 427-446; (chines.; engl. Abs.).

Hill, D. 1938-1941. A monograph on the Carboniferous rugose corals of Scotland. - The Palaeontographical Society [Monographs]: 1-213, 3 figs., 11 pls. [91 (for 1937, 412): $1-78$, pls. $1-2,1938 ; 92$ (for 1938, 416): 79-114, pls.3-5, 1939; 94 (for 1940, 419): 115-204, pls.6-11, 1940; 95 (for 1941, 424): 205-213, 1941]; London.

Korn, D. 1999. Famennian Ammonoid Stratigraphy of the Ma'der and Tafilalt (Eastern Anti-Atlas, Morocco). Abhandlungen der Geologischen Bundesanstalt 54: 147-179; Wien.

Korn, D., Klug, C. \& Reisdorf, A. 2000. Middle Famennian ammonoid stratigraphy in the Amessoui Syncline (Late Devonian; eastern Anti-Atlas, Morocco). - Travaux de l'Institut Scientifique (Université Mohammed V Agdal), série Géologie \& Géographie Physique 20: 69-77; Rabat.

Kullmann, J. (1997): Rugose corals in non-reef environments - the case of the Cyathaxonia fauna. - Boletín de la Real Sociedad Española de Historia Natural, Sección Geológica 92 (1-4): 187-195.

Lecointre, G. 1926. Recherches Géologiques dans la Meseta Marocaine. - Mémoires de la Société des Sciences Naturelles du Maroc 14: 1-158.

Liao Wei-hua \& Cai Tu-ci 1987. Sequence of Devonian rugose coral assemblages from northern Xinjiang. - Acta Palaeontologica Sinica 26 (6): 689-707; (chines.; engl. Rés.).

Lin Bao-yu \& Xu Shao-chun 1994. Late Devonian to Tournaisian rugose corals from South China and palaeontological events. - Courier Forschungsinstitut Senckenberg 172: $23-33$.

Oliver, W. A. jr. 1992. Corals from the Turkey Creek Limestone (Lower Devonian), Southern Oklahoma. - Bulletin, Oklahoma Geological Survey 145: 137-159.

Onoprienko, Yu. I. 1979. Novye rugozy iz perekhodnykh otlozheniy mezhdu devonom i karbonom Omolonskogo massiva. [New Rugosa findings in the Devonian/Carboniferous transitional deposits of the Omolon massif]. XIV Tikhookeanskiy Nauchnyy Kongress SSSR Khabarovsk avgust 1979; putevoditel' nauchnoy ekskursii po turu IX (biostratigrafiya i fauna pogranichnykh otlozheniy devona i karbona), prilozhenie $\mathbf{3}$ [Pacific Science Congress
USSR Khabarovsk august 1979; field excursion guidebook for tour IX (biostratigraphy and fauna of the Devonian-Carboniferous boundary deposits), supplement 3]; Coelenterata (Onoprienko, Yu. I., ed.): $1-152,32$ pls (3-73, pls 1-19); Magadan.

Poty, É. 1984. Rugose Corals at the Devonian-Carboniferous boundary. - Courier Forschungsinstitut Senckenberg 67: $29-35$.

Poty, É. \& Xu Shao-chun 1996. Rugosa from the DevonianCarboniferous transition in Hunan, China. - Mémoires, Institut Géologique de l'Université Catholique de Louvain 36: 89-139.

Różkowska, M. 1969. Famennian Tetracoralloid and Heterocoralloid fauna from the Holy Cross Mountains (Poland). - Acta Palaeontologica Polonica 14 (1): 1-187.

Sorauf, J. E. 1992. Late Devonian (Famennian) Rugose Coral Fauna of the Percha Shale of Southwestern New Mexico. -- Journal of Paleontology 66 (5): 730-749.

Sorauf, J. E. \& Pedder, A. E. H. 1986. Late Devonian rugose corals and the Frasnian-Famennian crisis. - Canadian Journal of Earth Sciences 23 (9): 1265-1287.

Soto, F. \& Liao Wei-hua 2002. Laccophyllidae Grabau, 1928 (Rugosa) from the Hongguleleng Formation (Devonian: Lower Famennian) at the northwestern margin of Junggar Basin (Northern Xinjiang, NW China). In (Schröder, S., Löser, H. \& Oekentorp, K., eds). Contributions to Coral Research. Commemorative volume to celebrate Dr. Dieter Weyer's 65th birthday. Coral Research Bulletin, 7: 209-220; Dresden.

Soto, F. \& Lin Bao-yo 1997. Biostratigraphic and biogeographic affinities of famennian rugose corals in the Dzungar-Hinggan Basin (Northern China). In (Avlar, H., Poll, K. \& Strauch, F., eds). Beiträge zur Korallenforschung und Museumsarbeit. Festschrift zum 60. Geburtstag von Prof. Dr. Klemens Oekentorp). - Coral Research Bulletin, 5: 239-246; Dresden.

Soto, F. \& Lin Bao-yu 2000. Corales rugosos de la Formación Honggulelong (Fameniense) en el N de Sinkiang (NO de China). - Geobios 33 (5): 527-541.

Termier, G. \& Termier, H. 1950. Paléontologie Marocaine. II. Invertébrés de l'ère primaire. 1. Foraminifères, spongiaires et coelentérés. - Notes et Mémoires Service Géologique Division des Mines et de la Carte Géologique, Protectorat République Francaise au Maroc 73: 1-220; Paris.

Tourneur, F. 1994. News and Views. Belgium. - Fossil Cnidaria and Porifera, Newsletter, International Association for the Study of Fossil Cnidaria and Porifera (International Palaeontological Association), 23 (1.1): 3-4; Münster (Forschungsstelle für Korallenpaläozoologie, Geologisch-Paläontologisches Institut und Museum, Westfälische Wilhelms-Universität).

Vasilyuk, N. P. 1970. Tselenteraty zony etren' Evrazii. [Etroeungtian Coelenterata of Eurasia]. In Kaljo, D. L. (ed.): Zakonomernosti rasprostraneniya paleozoyskikh korallov SSSR. Trudy 2. Vsesoyuznogo simpoziuma po izucheniyu iskopaemykh korallov SSSR, 3. - Institut Geologii i Geofiziki, Sibirskoe Otdelenie, Akademiya Nauk SSSR; 1-104, figs., tabs. (94-99); Moskva (Izdatel'stvo Nauka).

Wang Cheng-yuan 1987. On the age of Cystophrentis Zone. - Journal of Stratigraphy 11 (2): 120-125; Beijing (chines.; engl. Abs.).

Weyer, D. 1973. Über Protozaphrentis Yü, 1957 (Anthozoa Rugosa, Mittelordoviz). - Paläontologische Abhandlungen, Abteilung A, Paläozoologie 4 (4): 695-706; Berlin.

- 1984. Neaxon cheilos n.sp. aus dem Unterfamenne von Schleiz im Thüringer Schiefergebirge (Anthozoa, Rugosa; Oberdevon). - Abhandlungen und Berichte für Naturkunde und Vorgeschichte (Kulturhistorisches Museum Magdeburg), [for 1982], 12 (5): 3-16, 77-83.

- 1995. Heterocorallia aus Famenne-Cephalopodenkalken im Rheinischen Schiefergebirge und Tafilalt. - Abhand- 
lungen und Berichte für Naturkunde (Museum für Naturkunde Magdeburg) 18: 103-135.

- 1997. News about Famennian Heterocorallia in Germany and Morocco. - Boletín de la Real Sociedad Española de Historia Natural, Sección Geológica 91 (1-4): 145-151.

- 2000. Revision der Gattung Czarnockia Różkowska 1969 (Anthozoa, Rugosa; Oberdevon). - Abhandlungen und Berichte für Naturkunde, Museum für Naturkunde Magdeburg 21: 75-107 (dated 1999, issued 16. 3. 2000).

- 2001. Muenstraia, ein neues Rugosa-Genus (Anthozoa) aus dem Obersilur und Unterdevon. - Mitteilungen aus dem Museum für Naturkunde in Berlin, Geowissenschaftliche Reihe 4: 71-82.

Wu Wang-shi, Zhao Jia-ming \& Jiang Shui-gen 1981. Corals from the Shaodong Formation (Etroeungt) of South China. - Acta Palaeontologica Sinica 20 (1): 1-14; (chines.; engl. Abs.)

Yu Jian-zhang [YÜ C. C.] 1931. The Correlation of the Fengningian System, the Chinese Lower Carboniferous, as based on coral zones. - Bulletin of the Geological Society of China 10: $1-30$. 